\title{
THEORETICAL STUDIES IN HADRONIC AND NUCLEAR PHYSICS
}

\author{
A Progress Report for Research Supported By \\ the U.S. Department of Energy
}

Submitted July 1994

Principal Investigator: Dr. Thomas D. Cohen

Co-Principal Investigator: Dr. Manoj K. Banerjee

Period of Time This Report Covers: December 1, 1993 - June 30, 1994

Recipient Organization: University of Maryland

Department of Physics

College Park, Maryland 20742-4111

DOE Award No.: DE-FG02-93ER-40762

Budget Period: December 1, 1993-November 30, 1994

Estimated Unexpended Funds: $\$ 33,324$

\section{DISCLAIMER}

This report was prepared as an account of work sponsored by an agency of the United States Government. Neither the United States Government nor any agency thereof, nor any of their employees, makes any warranty, express or implied, or assumes any legal liability or responsibility for the accuracy, completeness, or usefulness of any information, apparatus, product, or process disclosed, or represents that its use would not infringe privately owned rights. Reference herein to any specific commercial product, process, or service by trade name, trademark, manufacturer, or otherwise does not necessarily constitute or imply its endorsement, recommendation, or favoring by the United States Government or any agency thereof. The views and opinions of authors expressed herein do not necessarily state or reflect those of the United States Government or any agency thereof.

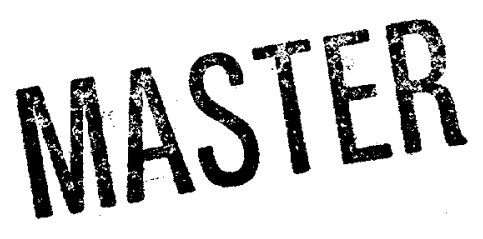




\section{DISCLAIMER}

Portions of this document may be illegible in electronic image products. Images are produced from the best available original document. 


\section{Contents}

I Introduction and Administrative Overview 3

A Research Progress ........................ 3

B Culture of the Maryland Nuclear Theory Research Group . . . . . . . . . . 4

C University's Distinguished Nuclear Theory Visitors Program . . . . . . . . 4

D Computer Support of Nuclear Theory Research . . . . . . . . . . . . . 4

E Nuclear Theory Faculty . . . . . . . . . . . . . . . . 5 5

F Cohen's Presidential Young Investigator Award and NSF Travel Grant . . . 5

G Long-Term Visitors with Independent Support and Local Research Affiliated

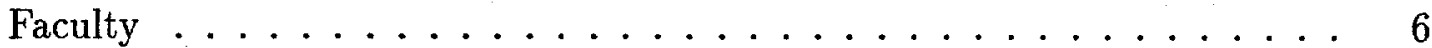

H Postdoctoral Researchers . . . . . . . . . . . . . . . . 6

I Graduate Research Assistants . . . . . . . . . . . . . . . 6

J Nuclear Theory Personnel Roster $\ldots \ldots \ldots \ldots \ldots$. . . . . . . 7

II Hadrons in Nuclei and Nuclear Matter $\quad 11$

A Nucleon in Nuclear Matter . . . . . . . . . . . . . . . . . . . . . . . 11

B A New Approach to Dense Matter in Chiral Quark Models . . . . . . . . . 13

C Quantum Mechanical Isospin Correlations and Disoriented Chiral Condensates 14

D General Results On Vanishing Chiral Condensates For Finite Density Nuclear Matter . . . . . . . . . . . . . . . . 14

E A Toy Model of Color Transparency . . . . . . . . . . . . . . 15

III Hadron Physics $\quad 16$

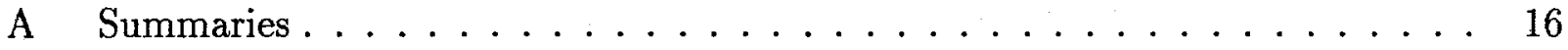

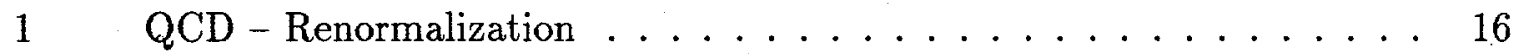

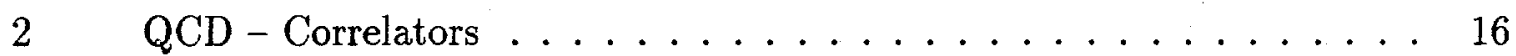

$3 \quad$ Perturbative QCD $\ldots \ldots \ldots \ldots \ldots \ldots \ldots \ldots$

4 Heavy Quark Physics . . . . . . . . . . . . . 17

$5 \quad$ QCD Sum Rules $\ldots \ldots \ldots \ldots \ldots \ldots \ldots \ldots$

$6 \quad$ QCD Based Models $\ldots \ldots \ldots \ldots \ldots \ldots \ldots$

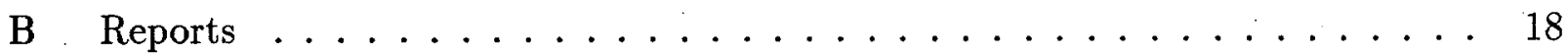

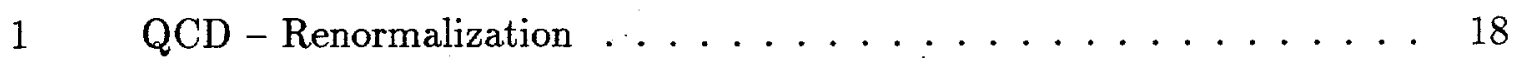

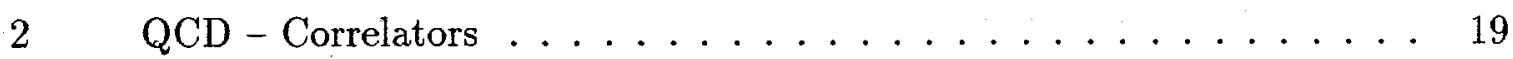

$3 \quad$ Perturbative QCD $\ldots \ldots \ldots \ldots \ldots$

4 Heavy Quark Physics . . . . . . . . . . . . . . . . 22 
5. QCD Sum Rules . . . . . . . . . . . . . . 22

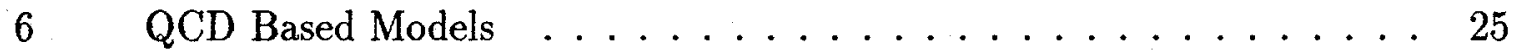

IV Relativistic Dynamics in Quark, Hadron and Nuclear Physics 29

A Low-Energy Theorem for Scalar Interactions of a Composite Spin-1/2 System . 31

B Relativistic Analysis of the Deuteron and Elastic Electron-Deuteron Scattering 35

C Quark-Antiquark Bound States Based on Two-Body Dirac Equation . . . . . 36

D Quasipotential Approach to the Relativistic Three-Body Problem . . . . . . 38

V Heavy Ion Dynamics and Sharp Lepton Pairs $4 \mathbf{4 1}$

A The Composite $\left(Q_{0}\right.$ ?) Particle Scenario and the Generalized "Sharp Lepton". Problem . . . . . . . . . . . . . . . . . . . . . 41

1 Recent Expansion of " $\left(e^{+} e^{-}\right)$-Puzzle" to the "Sharp Lepton Problem" 41

(a) Sharp Electrons from $\left(\beta^{+}+\right.$Atom) Expand the Range of Relevant Data . . . . . . . . . . . . . . . 41

(b) Sakai's Sharp Electrons Stringently Constrain the Originating Decay Process . . . . . . . . . . . . . . . . 41

(c) Upper Bound on Break-up Lifetime of Supercomposite Molecule . 42

(d) Data of $\left(e^{+} e^{-}\right)$-Puzzle and Sharp $\beta^{+}$Leptons Combine Into Single Expanded "Sharp Lepton Problem" . . . . . . . . . . . . . 42

2 Previous Successes of the Composite Particle Scenario for Heavy Ion $\left(e^{+} e^{-}\right)$Puzzle . . . . . . . . . . . . . . . . . 42

3 New Interest in Bhabha Scattering from Heavy Atoms; No Constraints on Particle Lifetime from Bhabha Data on Light Atom Targets . .

4 How Does $\left(Q_{0}\right.$ ?) Impinge Upon High Precision Quantum Electrodynamics? . . . . . . . . . . . . . . . . . . 43

$5 \quad$ Further Study of "Sharp Lepton Problem" . . . . . . . . . . . . . 44

B Permeation Current and Observed $\bar{N}, \bar{Z}$ Drifts in Heavy Ion Collisions . . . . 44

$\begin{array}{ll}\text { VI Research Publications: 1993-(July) } 1994 & 46\end{array}$ 


\section{Introduction and Administrative Overview}

Research in the Maryland Nuclear Theory Group focusses on problems in four basic areas of current relevance. They form the major divisions of the progress report summarized in four sections as follows.

- Hadrons in Nuclei and Nuclear Matter

- Hadron Physics

- Relativistic Dynamics in Quark, Hadron and Nuclear Physics

- Heavy Ion Dynamics and Sharp Lepton Pairs

\section{A. Research Progress}

Under Hadrons in Nuclei and Nuclear Matter we research the ways in which the properties of nucleons and mesons are modified in the nuclear medium. Research progress is reported on a number of topics in this general area, including studies of the role of chiral symmetry for finite density or temperature nuclear matter, the use of QCD sum rules to describe baryons in nuclear matter, and color transparency. In the general field of Hadron Physics broad progress included studies of perturbative QCD, heavy quark physics, QCD sum rules, and QCD-based models. Notable progress was also achieved in Relativistic Dynamics in Quark, Hadron, and Nuclear Physics, where an explicit model of composite particles shows how the z-graph physics (which is an essential part of Dirac phenomenology) comes about. In addition, calculations of elastic electron-deuteron scattering based on two-body relativistic dynamics and meson exchange currents were completed, as were studies of quarkanti-quark bound states based on a relativistic quark model. Progress is also reported on the relativistic few-body problem. In the area of Heavy Ion Dynamics and Sharp Lepton Pairs, work continues on the Composite Particle Scenario for the "Sharp Lepton Problem". In particular, the scenario can now encompass the anomalous sharp leptons reported from positron irradiation of heavy neutral atoms, establishing such irradiations as an alternative experimental window to the heavy ion experiments.

An overview presents the relevant philosophy and context of each of the four main segments of this report. More detailed reports addressing each specific topic can be located by the Table of Contents. 


\section{B. Culture of the Maryland Nuclear Theory Research Group}

The Maryland Nuclear Theory program is broadly based with several separate and independently evolving collaborations among the members of the group and their colleagues from other institutions. The interests of the group are strongly directed towards fundamental questions of long-term interest.

The group atmosphere is open, informal, and highly interactive, in part due to the custom of daily discussions at the group lunch. A weekly seminar is held where invited visitors present recent research accomplishments. The seminar and visitor programs provide a rich atmosphere for postdoctoral associates and students. We try to encourage new research questions and new research methods, and to maintain an internal process of constructive criticism to help keep our standards high. We believe that the resulting program is vigorous and exciting, and that as a result, the quality of the graduate students, postdoctoral researchers, and visiting faculty who join our group is excellent and their experiences with us are productive.

\section{University's Distinguished Nuclear Theory Visitors Program}

Special funding by the University of Maryland is sustaining a Distinguished Visitors Program in Nuclear Theory during the three-year period of the present DOE grant. Distinguished visitors are invited to spend significant periods of time at Maryland with funding of $\$ 25,000$ per year provided by the Department of Physics and the College of Computers, Mathematics and Physical Sciences of the University. This new program replaces support previously received from the Center for Theoretical Physics which was discontinued due to substantial cutbacks in the University budget. The allocation of scarce University resources to the new Nuclear Theory Distinguished Visitors Program is a clear signal of the healthy support for nuclear theory at the University of Maryland.

The Distinguished Visitors Program is already serving its purpose of bringing leading nuclear researchers to Maryland. We expect to have a mix of longer and shorter term visitors including sabbatical visitors in some years. We are considering a Special Year focussing on a single topic at the interface of nuclear and particle physics. As a bonus, our Distinguished Visitors Program has led to the involvement of some of our Department's particle physics faculty in topics of mutual interest, thereby enhancing constructive interaction between nuclear and particle theory.

\section{Computer Support of Nuclear Theory Research}

Our computing environment consists of a DECStation cluster with file service provided by a DECServer $5000 / 240$ with a $5 \mathrm{~Gb}$ disk, one of three DECstation $5000 / 240$ 's currently in the UNIX system. A second of the DECStation 5000's with $40 \mathrm{Mb}$ of fast memory provides 
compute service for large jobs. Five DECStation 3100's, seven DECStation 2100's, and a postscript printer round out the cluster.

System management expertise is provided by the University's Computer Science Center. A long-term arrangement for system support services for UNIX clusters is now being implemented. We expect support from the University's Computer Science Center to fulfill the group's current needs. The Department of Physics has hired a Coordinator for Computer Systems and Networking who also provides assistance.

In the next year, we plan to upgrade the system with a faster computer server, and to purchase one new DEC Alpha machine, and a postscript printer.

\section{E. Nuclear Theory Faculty}

The Nuclear Theory Group consists of five regular faculty members (Profs. M. K. Banerjee, T. D. Cohen, J. J. Griffin, W. M. MacDonald, and S. J. Wallace). Professor Stephen Wallace is now the Chairman of the Department of Physics and receives no salary from the grant. He continues to maintain an active research program and receives travel, visitor and secretarial support in connection with his research program. Professor William MacDonald, who is Director of Computer Services for the Physics Department, draws no salary from the contract; however, he occasionally receives some incidental support for his nuclear physics research activities.

Professors Thomas Cohen and James Griffin will also be partially supported by research sabbatical leave during the coming academic year.

Professor Edward Redish is now devoting all of his time to separately funded projects in innovative physics education, and thus is no longer formally part of the Nuclear Theory group's research activities.

\section{F. Cohen's Presidential Young Investigator Award and NSF Travel Grant}

Professor Cohen holds a Presidential Young Investigator Award which commenced in the Fall of 1990. This is a five-year award, which continues through the Spring of 1995. It provides Dr. Cohen with $\$ 25,000$ per year in research support.

In the Fall of 1993, Prof. Cohen was also awarded a three-year travel grant under the NSF Eastern Europe Program to allow collaborations between nuclear theorists of the University of Maryland and the Institute of Nuclear Physics in Cracow, Poland. This grant provides $\$ 6300$ per year travel support through 1996 . 


\section{G. Long-Term Visitors with Independent Support and Local Research Affiliated Faculty}

Dr. Marina Nielsen, Assistant Professor from the Univ. of São Paulo, Brazil, has been here on sabbatical leave for two years (through October 1994). She is supported by a fellowship granted by the Fundação de Amparao à Pesquisa do Estado de São Paulo, and is collaborating with Drs. Banerjee, Cohen, Forkel, Jin and Milana.

Dr. Hiroshi Ito of George Washington University continues close connections with the group throughout the year.

\section{H. Postdoctoral Researchers}

The Nuclear Theory Group currently has four postdoctoral researchers supported by our DOE grant. Dr. Hilmar Forkel will be leaving in November 1994 to accept a position at the European Center for Theoretical Studies in Trento, Italy. Dr. Hung Jung Lu will complete his two-year appointment in the Fall of 1994 . He will leave to accept a position at the University of Arizona. Dr. David Griegel will continue with us for the second year of his two-year appointment. Dr. Joseph Milana has accepted our offer of a one-year continuation of his postdoctoral position at Maryland. Two new postdoctoral research associates will join the group in September 1994: Drs. Yasuo Umino from NIKHEF-K, Amsterdam, and Siwen Wang from Carnegie Mellon University, Pittsburgh.

Dr. Xuemin Jin, who was supported under Dr. Cohen's P.Y.I. grant during the last year, has accepted a position at TRIUMF, Vancouver beginning September 1994.

\section{Graduate Research Assistants}

The group currently supports three students as Graduate Research Assistants engaged in Ph.D. research. C. Edward Bell completed his doctorate in the Summer of 1993 and continued his research with Prof. Wallace as a Faculty Research Assistant until January 1994. Myunggyu Kim is expected to complete his research during the coming academic year.

A list of Nuclear Theory Group personnel is given below: 


\section{J. Nuclear Theory Personnel Roster}

\section{Nuclear Theory Group Personnel (Dec. 1993-Nov. 1994)}

Faculty

Manoj K. Banerjee

Thomas D. Cohen $\dagger$

James J. Griffint†

William M. MacDonald*

Stephen J. Wallace*
Visiting Faculty

Marina Nielsen*

Research Associates

Hilmar Forkel (thru 10/94)

David Griegel

Xuemin Jin* (thru 8/94)

Hung Jung Lu (thru 8/94)

Joseph Milana

Yasuo Umino (from 9/94)

Siwen Wang (from 9/94)

Faculty Research Assistant

C. Edward Bell (thru 1/94)

Research Graduate Assistants

Mountaga Aw

Paul Dulany

Myunggyu Kim

* No salary support from DOE grant.

†On sabbatical leave July 1994-Sept. 1995.

††On sabbatical leave Aug. 1994-Jan. 1995.

The Group's activities are supported by one full-time secretary (Loretta Robinette). The Department of Physics provided $\$ 3600$ for secretarial support in 1993-94 academic year.

In addition to the Distinguished Visitors Program described above, we have long sustained an active Visitors Program to insure our group's ongoing contact with leading theorists, and to support our seminar program, our research collaborations, and the interviewing of postdoctoral candidates.

We list below all the visitors of the Nuclear Theory Group during 1993-1994. The * indicates the visit was under the auspices of our Distinguished Visitors Program. 


\section{3}

Mr. Ubirajara van Kolck, Univ. of Texas at Austin: 2 days (1/12-13/93)

* Dr. Carl Shakin, Brooklyn College of CUNY: 4 days (1/12-15/93)

Dr. Avraham Rinat, Weizmann Institute, Rehovot, Israel: 2 days (1/14-15/93)

Dr. Iosef Zhitnitsky, Columbia University, New York: 4 days (1/19-22/93)

Dr. Sadhan Adhikari, Inst. de Fisica Teorica, Sao Paulo: 4 days (1/27-30/93)

Dr. Wei Lin, Univ. of Washington, Seattle: 2 days (2/1-2/93)

Mr. Hua-Bin Tang, Indiana University: 2 days (2/9-10/93)

Dr. William Greenberg, Univ. of Washington, Seattle: 2 days (2/17-18/93)

Dr. Dirk Walecka, CEBAF and College of William and Mary: 1 day $(2 / 19 / 93)$

Mr. Patrick Labelle, Cornell University, Ithaca: 2 days (2/24-25/93)

Dr. Vijay Pandharipande, Univ. of Illinois at Urbana: 2 days (3/10-11/93)

Mr. Sen-Ben Liao, Massachusetts Inst. of Technology: 2 days (3/16-17/93)

Dr. Peter Tandy, Kent State University: 2 days (3/24-25/93)

Dr. Charles Benesh, Los Alamos National Lab, New Mexico: 4 days (4/1-4/93)

Mr. Chungsik Song, Univ. of Minnesota, Minneapolis: 3 days (4/6-8/93)

Dr. Suzhou Huang, Univ. of Washington, Seattle: 4 days (4/20-23/93)

* Dr. Andrew Jackson, SUNY, Stony Brook: 3 days (4/27-29/93)

Dr. Mark Bolsterli, NSF and Los Alamos National Lab: 1 day (5/5/93)

Dr. Daniel Bes, Atomic Energy Commission, Buenos Aires: 2 weeks (5/25-6/7/93)

Dr. Dinesh K. Srivastava, Variable Energy Cyclotron Ctr., Calcutta: 3 days (6/10-13/93)

Dr. Alan Sommerer, Iowa State University: 2 days (6/14-16/93)

* Dr. Ernest Henley, University of Washington: 2 days (6/15-16/93)

Dr. Bojan Golli, Inst. Josef Stefan, Ljubljana, Slovenia: 5 days (7/5-9/93)

* Dr. Boris Ioffe, Inst. for Theor. \& Exp. Physics, Moscow, Russia: 2 weeks (7/15-31/93)

Dr. Pankaj Jain, University of Kansas: 2 days (7/15-16/93)

Dr. Baha Balantekin, University of Wisconsin: 2 day (8/2-3/93)

Dr. Gunther Piller, University of Regensburg, Germany: 3 days $(8 / 2-4 / 93)$

Dr. Richard Furnstahl, Ohio State University: 2 days (9/23-24/93)

Dr. Carl Carlson, College of William \& Mary: 2 days (9/30-10/1/93)

Dr. Sean Gavin, Brookhaven National Lab: 1 day (10/15/93)

* Dr. John Donoghue, University of Massachusetts: 3 days (10/22-24/93)

Dr. Mira Dey, Calcutta, India: 3 days (10/26-28/93)

Dr. Jishnu Dey, Calcutta, India: 3 days (10/26-28/93)

Dr. Alfred Mueller, Columbia University: 1 day (11/5/93)

Dr. Johan de Swart, University of Nijmegen, The Netherlands: 1 day $(11 / 8 / 93)$ 
Dr. Scott Pratt, Michigan State University: 2 days (11/11-12/93)

Dr. Matthias Lutz, University of Washington: 2 days (11/15-16/93)

Dr. Jose Goity, CEBAF, Newport News: 2 days: (11/18-19/93)

Dr. Oriol Bohigas, IPN, Orsay, France: 1 day (12/3/93)

Mr. Thomas Hyer, Stanford Linear Accelerator Ctr.: 1 day (12/6/93)

* Dr. Stanley Brodsky, Sanford Linear Accel. Ctr.: 5 days (12/6-10/93)

\section{4}

Mr. Chris Jarzynski, Lawrence Berkeley Lab.: 2 days (1/10-11/94)

Dr. Dharam Ahluwalia, Los Alamos Nat. Lab: 1 day (1/21/94)

Mr. Zheng-Kun Zhu, Drexel Univ.: 1 day (1/18/94)

Mr. Yohanes Surya, CEBAF \& College of William \& Mary: 1 day (2/4/94)

Mr. Chengqian Gong, Duke Univ.: 5 days (2/10-14/94)

Mr. Jonathan Bennett, Univ. of Wisconsin-Madison: 1 day (2/18/94)

Dr. Kenneth Wilson, Ohio State Univ.: 1 day $(2 / 22 / 94)$

* Dr. Avraham Rinat, Weizmann Inst.: 2 days (2/24-25/94)

Mr. Jacek Szweda, Brooklyn College: 2 days (2/27-28/94)

Dr. Hsien-Chi Jean, Florida State Univ.: 1 day (3/4/94)

Dr. Jitendra Parikh, Physical Research Lab., Ahmedabad, India: 3 days (3/7-9/94)

* Dr. John Tjon, Univ. of Utrecht, The Netherlands: 3 days (3/14-16/94)

* Dr. Brian Serot, Indiana Univ.: 4 days (3/15-18/94)

Dr. Robert Perry, Ohio State Univ.: 1 day (3/25/94)

Dr. Rocco. Schiavilla, CEBAF and Old Dominion Univ.: 1 day (4/1/94)

* Dr. Miklos Gyulassy, Columbia Univ.: 1 day (4/7/94)

* Dr. John Negele, Massachusetts Inst. of Tech.: 1 day (4/22/94)

Dr. Adam Szczepaniak, North Carolina State Univ.: 1 day (4/25/94)

Dr. Roxanne Springer, Duke University: 1 day (4/29/94)

Dr. Yasuo Umino, NIKHEF-K, Amsterdam: 4 days (5/3-6/94)

Mr. Daniel Phillips, Flinders Univ., S. Australia: 3 days (5/10-13/94)

Dr. Wally Melnitchouk, Univ. of Regensburg, Germany: 4 days (5/21-25/94)

Dr. Gastao Krein, Inst. de Fisica Teorica, São Paulo, Brazil: 4 days (5/21-25/94)

Dr. Sadhan Adhikari, Inst. de Fisica Teorica, São Paulo, Brazil: 2 days (5/31-6/2/94)

Dr. Hanxin He, China Inst. of Atomic Energy, P.R.China: 3 days (6/15-18/94)

Dr. Mahir Hussein, Univ. of São Paulo, Brazil: 1 day (6/17/94)

Mr. Siwen Wang, Carnegie Mellon Univ., Pittsburgh, PA: 1 day (6/20/94) 


\section{Long-Term Visitors}

* Dr. John A. Tjon, University of Utrecht, The Netherlands: 7 weeks (3/15-4/30/93)

* Dr. Shmuel Nussinov, Tel-Aviv University, Israel: 3 weeks $(6 / 28-7 / 15 \& 8 / 9-13 / 93)$

* Dr. Dan-Olof Riska, University of Helsinki, Finland: 3 weeks (12/2-21/93)

* Dr. Gerald Brown, S.U.N.Y. at Stony Brook, NY: 4 weeks (5/9-6/3/94)

$\dagger$ Dr. Wojciech Broniowski, Inst. of Nuclear Physics, Krakow, Poland: 4 weeks (5/1-6/1/94)

* Dr. John A. Tjon, University of Utrecht, The Netherlands: 3 weeks (5/18-25 \& 6/1-11/94)

* Dr. Shmuel Nussinov, Tel-Aviv University, Israel: 6 weeks (7/1-8/15/94)

Frequent Local Visitor

Dr. Hiroshi Ito, George Washington University, Washington, DC

* Supported under the Distinguished Visitors Program.

† Supported by Dr. Cohen's Presidential Young Investigator's Award. 


\section{Hadrons in Nuclei and Nuclear Matter}

The general problem of how hadrons behave in the nuclear environment is at the core of nuclear physics. The nuclear theory effort at Maryland has addressed a number of problems in this general field by a wide range of techniques.

Much of this activity has been on the application of QCD sum rule techniques to describe hadrons propagating in nuclear matter. This has been part of an ongoing research program. The major progress in the past year has been the completion of a systematic study of nucleons in nuclear matter. The analysis has also been extended to the problem of $\Sigma$ hyperons in nuclear matter.

Another approach to this problem is via chiral confining model which has long been studied here. The effect of the nuclear environment is included via meson fields acquiring expectation values different from their vacuum values. Another approach to modeling hadrons in nuclear matter is the use of a hyperspherical unit cells to simulate finite nuclear density. This approach has been used to study various chiral models.

Several projects stressed the role of chiral symmetry for finite temperature or density nuclear matter. For example, we have addressed the issue of disoriented chiral condensates which have been postulated to form if hadronic matter above the chiral phase transition cools rapidly. We have shown that quantum mechanical isospin correlations between the condensate and the rest of the system can alter the distribution of the ratio charged to neutral pions emerging from the condensate. We have also addressed the general question of what happens when the chiral condensate, $\langle\bar{q} q\rangle$, vanishes due to a high density of nuclear matter. We have shown on the basis of current algebra that either chiral symmetry is restored or the pion has become anomalously light. Such an anomalously light pion may be interpreted as a precursor of s-wave pion condensation.

A final issue addressed in this section is the notion of color transparency. A very common formulation of the idea is that in a hard scattering event a "small wave packet" forms which interacts weakly with the nuclear environment. We have formulated a tractable atomic physics analog of the nuclear case and demonstrated explicitly both that the phenomena can occur and that the key point is not that the wave packet is small but that the overlap of the wave packet and the observed hadron is of a small spatial extent.

\section{A. Nucleon in Nuclear Matter}

We have continued our earlier work on the possible density dependence of meson-nucleon coupling constants and its effect on the properties of nuclear matter.

A scheme for a simple calculation has been described in an earlier paper [1]. The resulting density-dependent coupling constants were used by $\mathrm{J}$. Tjon in his relativistic nuclear matter 
program developed with Amorim [2]. The results are shown in Table 1. While the

\begin{tabular}{|l|l|l|l|l|}
\hline Model & Type & $\begin{array}{l}\rho \\
\text { in } \mathrm{fm}^{-3}\end{array}$ & $\begin{array}{l}k_{F} \\
\text { in } \mathrm{MeV}\end{array}$ & $\begin{array}{l}\text { E/A } \\
\text { in } \mathrm{MeV}\end{array}$ \\
\hline $\begin{array}{l}\text { Amorim } \\
\text { \& Tjon }\end{array}$ & & .0 .127 & 243 & -11.8 \\
\hline Toy & Quartic & 0.088 & 215 & -11.3 \\
\hline Toy & Pure Mass & 0.059 & 188 & -8.6 \\
\hline $\begin{array}{l}\text { Toy }+ \\
\text { 't Hooft }\end{array}$ & Quartic & 0.086 & 214 & -15.4 \\
\hline
\end{tabular}

Table 1: Results of relativistic nuclear matter calculations with density-dependent coupling constants. Columns 1 and 2 describe the basis of calculation. The next three columns list the values of density, Fermi momentum and $E / A$ at saturation.

results are not good, the nuclear matter is still bound. We were not surprised by the bad result. There were several points where the theory could be improved. Last year we have made two major improvements.

The first item is the improvement of the simple Toy model by the inclusion of the 't Hooft interaction [3]. The second was to recognize the role of the pion cloud in the coupling of the $\sigma$ and the $\rho$ mesons to the nucleon. The method of incorporating the role of the $\pi$ meson is illustrated with the example of $g_{\sigma N N}$. We write

$$
g_{\sigma N N}=g_{\sigma N N}^{(q)}+\xi\left(g_{\pi N N} / 2 M_{N}\right)^{2}
$$

where $g_{\sigma N N}^{(q)}$ describes the direct coupling of $\sigma$ to the quarks and the second terms describes the coupling to the pion cloud. The value of $g_{\sigma N N}^{(q)}$ and $g_{\pi N N} / 2 M_{N}$ in vacuum are taken from Ref. [3]. The value of $g_{\sigma N N}$ in vacuum is taken to be 8 . This fixes the constant $\xi$. The density dependence of $g_{\sigma N N}$ is determined with the help of known dependence of $g_{\sigma N N}^{(q)}$ and $g_{\pi N N} / 2 M_{N}$ on the $\sigma$ and the $\omega$ fields in the manner described in Ref. [1]. The Dirac and Pauli couplings of the $\rho$ meson, $g_{\rho N N}$ and $f_{\rho N N} / 2 M_{N}$ are treated in a similar manner.

Preliminary results of using these density-dependent coupling constants in nuclear matter calculations have been obtained by Tjon. These are shown in Table 1. The binding energy has improved considerably. The saturation density is almost the same as in the calculation with the Toy model. We are continuing our work, which is obviously needed. 
[1] M. K. Banerjee, Phys. Rev. C 45 (1992) 1359.

[2] A. Amorim and J. A. Tjon, Phys. Rev. Lett. 68 (1992) 772.

[3] M. Kim and M. K. Banerjee, Phys. Rev. C 48 (1993) 2035.

\section{B. A New Approach to Dense Matter in Chiral Quark Models}

The baryon structure and its modifications in nuclear matter become increasingly important at higher densities. Changing baryon properties will strongly influence the behavior of very dense matter, in particular close to the expected deconfinement and chiral restoration phase transitions. In the hypersphere approach, the matter is divided into cells with unit baryon number, and their interactions with the dense environment are modeled by space curvature. In the Skyrme model this approach reproduces the results of array calculations and, in particular, the chiral restoration phase transition at high densities almost quantitatively, and with greatly reduced calculational effort.

The generalization of this technique to quark-based hadron models [1],[2] induces a new, isoscalar gauge interaction for the quarks, which mediates interactions with the surrounding matter. The application of this method to the Nambu-Jona-Lasinio (NJL) model reproduces rather closely the density dependence found in the alternative chemical-potential approach, for the dynamical quark mass, the pion decay constant and the chiral order parameter. Furthermore, the description of valence-quark free cavities in the matter (related to a "bubble phase") becomes possible and the presence of explicit quark fields leads to new insights into the physical foundations of the approach.

The generalization of these results to finite temperature is a necessary step towards the physical conditions under which dense matter is produced in heavy-ion collisions. While the study of skyrmions on the hypersphere at finite temperature is complicated by fluctuations around the space-dependent soliton profile, these problems are absent in the NJL model. We thus use it for the first study of combined effects of finite temperature and baryon density in the hyperphere approach [3]. We discuss, in particular, the charateristic role of the temperature for the chiral phase structure and the critical density

(H. Forkel)

[1] H. Forkel, Phys. Lett. B 280 (1992) 5.

[2] H. Forkel, U. Md. preprint \#94-037, DOE/ER/40762-017 (1994).

[3] H. Forkel and A.D. Jackson, U. Md. preprint \#95-009, DOE/ER/40762-044 (1994). 


\section{Quantum Mechanical Isospin Correlations and Disoriented Chiral Conden- sates}

The idea of disoriented chiral condensates, introduced by Anselm [1] Bjorken [2] and others has been widely discussed in the context of heavy ion physics and perhaps in ultra-high energy hadronic collisions. A simple semiclassical analysis of such condensates (assuming a single large domain) suggests that the ratio, $R=$ number of neutral pions to total pions emerging from the condensate (at low transverse momentum), is given by $P(R)=1 /\left(2 R^{1 / 2}\right)$. We have shown that this derivation implicitly assumes that the condensate carries no net isospin [3]. Correlations in isospin between the pions in the condensate and the other hadrons in the problem allow the condensate to carry net isospin which alter $P(R)$. We have shown that moments of the $P(R)$ distribution can be related to expectation values of $I^{2 n} / N^{2 n}$ where $n$ is an integer, $\mathrm{I}$ is the isospin carried by the condensate, and $\mathrm{N}$ the total number of pions in the condensate. For example, for a single large domain of condensate, $\int d R P(R) R^{2}=1 / 5-$ $1 / 15\left\langle I^{2} / N^{2}\right\rangle$. The expectation values of $I^{2} / N^{2}$ can, in principle, be extracted experimentally from the distribution of $R_{c}=\left(N_{+}-N_{-}\right) N$ where $N_{+}$is the number of low $P_{T} \pi^{+}$'s emerging from the condensate. We have also shown that effects of these isospin correlations (relative to the semiclassical result) on $P(R)$ is concentrated in the endpoint regions near $R=0$ and $R=1$.

(T. D. Cohen, M. K. Banerjee, M. Nielsen and X. Jin)

[1] A. A. Anselm, Phys. Lett B217 (1989) 169.

[2] J. D. Bjorken, Acta Physica Polonica B23 (1992) 637.

[3] T. D. Cohen, M. K. Banerjee, M. Nielsen, X. Jin, U. Md. preprint \#94-101, DOE/ER/40762-031, to be published in Phys. Lett. B.

\section{General Results On Vanishing Chiral Condensates For Finite Density Nuclear Matter}

We have used current algebra to study the general problem of chiral symmetry in nuclear matter. We have demonstrated the chiral condensate $\langle\bar{q} q\rangle$ is negative semi-definite. If at some density the condensate goes to zero than either chiral symmetry is restored or the pion is anomalously light. By anomolously light, we mean that $m_{\pi}^{2} \sim m_{q}^{2}$ rather than $m_{\pi}^{2} \sim m_{q}$ as one has for a nonvanishing chiral condensate. This is significant in the context of s-wave pion condensation since pion condensation occurs when the pion goes massless. Thus, a vanishing chiral condensate without chiral restoration is an indication that one is close to s-wave pion condnesation.

(T. D. Cohen, W. Broniowski (Crackow)) 


\section{E. A Toy Model of Color Transparency}

We have studied a analog of color transparency in an artififical atomic system. The key advantage of this model is its computational tractablity relative to the nuclear physics case. The model consists of a nonrelativistic hydrgenlike atom (with equal constituent masses), the analog of a nucleon, confined to a well via an optical potential, which is the analog of the rest of the nucleus. The imaginary part of the potential is chosen so that the absorption is proportional to the quantum mechanical $r^{2}$ operator of the internal atomic wavefunction (which is consitent with the genral argument for color transparency); the mean-free path of a hydrogen atom in the medium described by the optical potential is chosen to be independent of energy to approximate the nuclear physics case. The process studied is exclusive electron scattering to a final state with a hydrogen atom in its ground state and free of the well. We find that at large momentum transfers we get transpareny-i.e. the cross section goes to the free space cross section. The key to obtaining transparency is that the overlap of the hydrogen atom's wave function after the hard scattering with the free hadron atom has a small spatial extent.

(T. D. Cohen, J. Schwarz and P. Lynch) 


\section{Hadron Physics}

The section on 'Hadron Physics' describes studies of structures of single hadrons and their interactions in free space. All the studies are either directly based on QCD or strongly motivated by it. There are 14 items in this report, divided into six groups. The report begins with paragraph summaries of the 14 items, arranged by groups. This is followed by more detailed reports, also arranged by groups.

\section{A. Summaries}

\section{1. $Q C D-$ Renormalization}

A novel approach has been developed by H. J. Lu and S. Brodsky (SLAC) in which different physical quantities calculated in perturbative QCD can be related to each other without renormalization scale or scheme ambiguity. The scales at which different quantities are evaluated have a commensurate relationship.

\section{2. $Q C D-$ Correlators}

H. Forkel has calculated baryon correlators supplementing the usual operator product expansion with contributions from 'direct' instantons (size $\leq 0.3 \mathrm{fm}$ ). The instantons make important contributions in the octet case and, as expected, smaller contributions in the decuplet case. In general, inclusion of instanton effects improve the agreement with the results of instanton-liquid simulations and lattice calculations.

$\mathrm{X}$. Jin and T. Cohen have studied zero momentum correlators of bosons containing one heavy and one light quark and find that these are expressible in terms of quark condensates. In turn, these yield model-independent relations among correlators which may serve as useful constraints in other areas, such as, lattice QCD, QCD sum rules, etc.

\section{Perturbative QCD}

J. Milana and Shmuel Nussinov (Tel Aviv) have completed the investigation of the decay process $J / \psi \rightarrow \pi^{+} \pi^{-}$as a source of pion form factor in the time-like region. They conclude that background processes, etc., do not introduce significant uncertainties. They extract the value $M_{J}^{2} F_{\pi}\left(M_{J}^{2}\right) \approx .9$.

Milana points out that possible nuclear dependence of the ratio $R=\sigma_{L} / \sigma_{T}$ can spoil the extraction of $Q^{2}$ dependence of the nuclear structure functions, $F_{2}^{A}\left(x, Q^{2}\right)$. Precision measurements of the variation of $R$ is needed to resolve the difficulty. His report at CEBAF on this problem and the need for further experiments was warmly received. 
Milana and Carl Carlson (William and Mary) have extended their earlier work on the decay of the $B$ meson into nearly all two-meson channels. Their results agree with experimental data. They find that the Isgur-Wise form factor is a dipole. They also conclude that heavy-quark symmetry may not be useful in the analysis of these decays.

The decay rate of $B \rightarrow K^{*} \gamma$ has been calculated by Milana and Carl Carlson (William and Mary). The penguin graphs (photon coming off a weak boson loop) give a branching ratio $\approx$ few $\times 10^{-5}$.

One expects that at LEP200, the future upgrade of the CERN electron-positron collider, there will be events with rapidity gaps. H. J. Lu has studied mechanisms of the class where lepton pairs annihilate into gauge bosons, $\gamma$ 's, $Z$ 's, etc, which then break up into quark pairs and find that these can generate gap events at observable rates.

\section{Heavy Quark Physics}

J. Milana and T. Cohen have developed a new method, based on a sum rule for semileptonic decays of the $B$ meson, to extract the CKM matrix element $\left|V_{\mathrm{bc}}\right|$. The method relies essentially on the much weaker assumption that the virtual $c \bar{c}$ pair content of the $B$ meson can be neglected.

\section{QCD Sum Rules}

H. Forkel , M. Nielsen and X. Jin are using the QCD sum rules to calculate the pion electromagnetic form factor. The novel feature of the project is that it includes the role of small-sized instantons which are known to play a crucial role in the pion correlator.

Jin , Nielsen and J. Pasupathy (Indian Inst. of Science, Bangalore) have calculated $\delta M_{N}$, the neutron-proton mass difference using the chirally even QCD sum rules. The result is in good agreement with the experimental value.

Forkel and Nielsen have included the role of instantons in the QCD sum rules for $\delta M_{N}$, the neutron-proton mass difference. The result of the chirally odd sum rule, which was off by an order of magnitude, improves significantly, while the previously successful chirally even sum rule is unaffected.

D. Griegel and T. Cohen have tested the results of the QCD sum rules for the nucleon mass and the $\sigma$ term against the well-established leading nonanalytic behavior of these quantities as functions of the current quark mass. They discover that the results of the QCD sum rules are not fully consistent with the required leading nonanalytic behavior and conclude that this may result in uncertainties of $\sim 100 \mathrm{MeV}$ in the results. 


\section{QCD Based Models}

Using their previously developed model H. Forkel, M. Nielsen, X. Jin and T. Cohen have estimated the strangeness vector form factors of the nucleon in the momentum region relevant for the planned experiments at MIT-Bates and CEBAF. The ingredients of the model are the kaon cloud contribution and vector meson dominance contribution coupled with $\omega-\phi$ mixing. They find that both Sachs form factors are dominated by the VMD contribution.

M. Kim and M. Banerjee have introduced configuration mixing in their chiral confining model. Configurations are constructed with spinors obtained in the mean field treatment of the Toy model. The following configurations are used: $\left(1 s \frac{1}{2}\right)^{3},\left(1 s \frac{1}{2}\right)^{2}\left(1 d \frac{3}{2}\right),\left(1 s \frac{1}{2}\right)^{2}\left(2 s \frac{1}{2}\right)$, $\left(1 s \frac{1}{2}\right)\left(1 p \frac{1}{2}\right)^{2},\left(1 s \frac{1}{2}\right)\left(1 p \frac{3}{2}\right)^{2}$, and $\left(1 s \frac{1}{2}\right)\left(1 p \frac{1}{2}\right)\left(1 p \frac{3}{2}\right)$. The hamiltonian, $H_{\text {Toy }}+H_{t H o o f t}$, is diagonalized in this space. Pionic effects are added perturbatively. The longstanding problem with $\left\langle r^{2}\right\rangle_{\text {neutron }}$ is removed.

\section{B. Reports}

\section{1. $Q C D-R e n o r m a l i z a t i o n$}

Commensurate Scale Relations in Quantum Chromodynamics [1]

H. J. Lu and S. J. Brodsky explore a novel approach to study perturbatively calculable quantities in QCD [1]. In this approach, physical observables such as the annihilation ratio $R_{e^{+} e^{-}}$, the heavy quark potential, and radiative corrections to structure function sum rules can be related to each other without renormalization scale or scheme ambiguity. The commensurate scale relations connecting the effective charges for observables $A$ and $B$ have the form $\alpha_{A}\left(Q_{A}\right)=\alpha_{B}\left(Q_{B}\right)\left(1+r_{A / B} \frac{\alpha_{B}}{\pi}+\cdots\right)$, where the coefficient $r_{A / B}$ is independent of the number of flavors $f$ contributing to coupling constant renormalization. The ratio of scales $Q_{A} / Q_{B}$ is unique at leading order and guarantees that the observables $A$ and $B$ pass through new quark thresholds at the same physical scale. We also show that the commensurate scales satisfy the renormalization group transitivity rule which ensures that predictions in PQCD are independent of the choice of an intermediate renormalization scheme $C$. In particular, scalefixed predictions can be made without reference to theoretically-constructed renormalization schemes such as $\overline{\mathrm{MS}}$.

The generalization of the BLM procedure to higher order assigns a different renormalization scale for each order in the perturbative series. The scales are determined by a systematic resummation of running coupling constant effects. The application of this procedure to relate known physical observables in QCD gives surprisingly simple results. In particular, we find that up to light-by-light type corrections, all terms involving $\zeta_{3}, \zeta_{5}$ and $\pi^{2}$ in the relation between the annihilation ratio $R_{e^{+} e^{-}}$and the Bjorken sum rule for polarized electroproduc- 
tion are automatically absorbed into the renormalization scales. The final series has simple coefficients which are independent of color: $\hat{\alpha}_{g_{1}}(Q)=\hat{\alpha}_{R}\left(Q^{*}\right)-\hat{\alpha}_{R}^{2}\left(Q^{* *}\right)+\widehat{\alpha}_{R}^{3}\left(Q^{* * *}\right)$, where $\hat{\alpha}=\left(3 C_{F} / 4 \pi\right) \alpha$. The coefficients in the commensurate scale relation can be identified with those obtained in conformally-invariant gauge theory.

(H. J. Lu and S. Brodsky (SLAC))

[1] S. J. Brodsky and H. J. Lu, U. Md. Preprint \#94-135, DOE/ER/40762-036 (1994).

\section{2. $Q C D-$ Correlators}

\section{Instanton-corrected Operator Product Expansion}

The conventional, nonperturbative operator product expansion (OPE), on which e.g. the QCD sum rule approach is based, neglects contributions from nonperturbative short-distance physics, originating mainly from small-scale ("direct") instantons. Phenomenological estimates, instanton-liquid simulations and lattice results indicate, however, that small-size instantons form an important component of the QCD vacuum.

To study their role and significance in baryon correlation functions, we calculate direct instanton corrections to the OPE in semiclassical approximation, taking all quark zero-mode contributions in the instanton background into account. This approximation to the correlator is expected to work well for distances up to the average separation between instantons.

We find significant contributions to the baryon octet correlators, and, as expected, smaller ones in the decuplet channel. We discuss their characteristic features and compare our results to the recent instanton-liquid and lattice data. The instanton corrections considerably improve the agreement between the anaytical, OPE based representation of the correlators and the results of the lattice and liquid simulations.

(H. Forkel)

\section{Perturbative $Q C D$}

Does $J / \psi \rightarrow \pi^{+} \pi^{-}$Fix the Electromagnetic Form Factor $F_{\pi}(t)$ at $t=M_{J / \psi}^{2} ?[1]$

This was work done in collaboration with Shmuel Nussinov while he was visiting the group last summer. It is a natural outgrowth of work contained in last year's progress report [2] addressing the viability of using the measured decay rate of the $J / \psi$ into $\pi^{+} \pi^{-}$to extract the electromagnetic form factor of the pion at large time-like invariant momentum. The dominant decay mode for this process is for the charm quarks in the $J / \psi$ to annihilate into a virtual photon, which then produces the pion pair. The work in last year's progress report was based on a perturbative QCD estimate of the background processes that could spoil this extraction, 
ending with the conclusion that "charmonium provides a good laboratory for extracting the pion's electromagnetic form factor at large $Q^{2}$ and that $M_{J}^{2} F_{\pi}\left(M_{J}^{2}\right) \approx .9$, although there may be other mechanisms (e.g.isospin violation in the strong interaction or the existence of some exotic bound state), that could yet spoil this result." The letter with Shmuel explicitly addresses these last deficiencies, demonstrating that they, too, are ignorable.

\section{(J. Milana and S. Nussinov (Tel Aviv))}

[1] J. Milana, S. Nussinov and M. G. Olsson, Phys. Rev. Lett. 71, 2553 (1993).

[2] R. Kahler and J. Milana, Phys. Rev. D 47, R3690 (1993).

\section{On the $A$ Dependence of $R=\sigma_{L} / \sigma_{T}$ and the $Q^{2}$ Dependence of Shadowing [1]}

In this work J. Milana points out the existence of a loophole in the usual analysis of the shadowing data created by the possibility of a nuclear dependence to $R=\sigma_{L} / \sigma_{T}$. A most counter-intuitive dependence in the differential cross section in the shadowing regime is shown to result from a higher-twist nuclear enhancement in $R$ as might arise due to fermi motion or other many-body, nuclear structure effects. Such a conjectured dependence, consistent with both the general theoretical framework of QCD and with existing data, severely complicates the unravelling from present measurement the corresponding dependence in $Q^{2}$ of the nuclear structure functions, $F_{2}^{A}\left(x, Q^{2}\right)$. Indeed, until precision measurements close this loophole, the extrapolations of the structure functions to either higher $Q^{2}$ or other processes (as is neccessary to address present data at FNAL or future experiments at RHIC) must be considered problematic. At a workshop this past April, Milana argued that CEBAF can make an important contribution to resolving this issue through systematic experimental determination of $R(A)$. This suggestion was warmly received by a number of experimentalists in the audience, including some here at Maryland, and we anticipate at least one experimental proposal as an outgrowth.

(J. Milana)

[1] J. Milana, Phys. Rev. C 49, 2820 (1994).

Systematics of Exclusive B-decays: a PQCD analysis [1]

The two-body exclusive decay modes of the $B$-meson are short distance events and can thus be addressed by perturbative QCD. In an earlier letter [2] the formalism for two-body mesonic decays was developed and applied to three illustrative cases: $B^{0} \rightarrow D^{-} D_{s}^{+}, B^{0} \rightarrow D^{-} \pi^{+}$and $B^{0} \rightarrow \pi^{-} \pi^{+}$. In this follow-up work a systematic analysis of nearly all the two-body, mesonic decays of the $\mathrm{B}$-meson was presented. Some of the results obtained were: 1) reasonably good accord with all experimentally observed rates; 2) the Isgur-Wise form factor is a dipole, 
however; 3) heavy-quark symmetry may not prove to be particularly useful as in those decays involving $D$-mesons, large corrections to heavy-quark symmetry are expected, appearing most prominently in the spin-flipped sector between $D$ and $D^{*}$ decay modes. Since these decays are as far from the zero recoil point as is kinematically possible, such corrections should not be particularly surprising, and indeed support recent lattice results for the heavy-meson decay constants, $f_{B}$ and $f_{D}$.

\section{(J. Milana and C. Carlson (William and Mary))}

[1] C. E. Carlson and J. Milana, Phys. Rev. D 49, 5908 (1994).

[2] C. E. Carlson, J. Milana, Phys. Lett. B301, 237 (1993).

Penguins Leaving the Pole: Bound State Effects in $B \rightarrow K^{*} \gamma[1]$

Applying the perturbative QCD methods developed above, the issue of bound-state effects in the recently observed, rare (penguin) decay $B \rightarrow K^{*} \gamma$ was investigated. Consistent with most analyses, it is shown that gluonic penguins, with photonic bremsstrahlung off a quark, could be safely ignored for now, contributing to the decay rate at only the few percent level. However, explicit off-shell $b$ quark effects normally discarded are found to be large in amplitude, although in the standard model accidents of phase minimize the effect on the rate. Using an asymptotic distribution amplitude for the $K^{*}$ and just the standard model, a branching ratio of a few $\times 10^{-5}$ was obtained, consistent with the observed rate. One diagram that had to be omitted for now was the double penguin, where both a photon and a gluon come out of a weak boson loop, as the effective vertex does not yet exist in the literature. While being the anticipated focus of a future study, its contribution can only improve our estimates, as it contributes strictly incoherently to the amplitude already calculated.

\section{(J. Milana and C. Carlson (William and Mary))}

[1] C. E. Carlson and J. Milana, WM-94-104 and U. Md. preprint \#94-129, DOE/ER/40762-033 (1994).

Rapidity Gap in Jet Events at LEP200

There has been an intense interest in the study of jet events containing rapidity gaps at particle colliders. The study of these events help to elucidate the nature of the fragmentation process by which short-distance partons materialize into long-distance hadrons. In two previous papers [1], the production of gap events at electron-positron colliders at $Z$ peak energy has been studied. In this work [2]. we analyze various perturbative mechanisms for the production of gap events at LEP200, the future upgrade of the electron-positron collider operating at CERN. These mechanisms are characterized by the annihilation of the initial leptons into two gauge bosons, which could be two virtual photons, one virtual photon and 
one $Z$, two $Z$ s, or two $W$ bosons. The gauge bosons subsequently decay into quark-antiquark pairs, generating the outgoing jets. For specific kinematic and color configurations, the jet events can contain a rapidity gap, i.e., a region in the longitudinal phase space with no final hadron particles. We point out that LEP200 offers an advantageous environment for the study of rapidity gap events, since the number of fake gap events due to random fluctuation of final hadrons is substantially reduced with respect to the present colliders operating at the $Z$-peak energy. We show that the described mechanisms give an observable rate. The experimental study of these events will provide important information on rapidity gap physics and also on the color flow during the fragmentation process.

(H. J.Lu)

[1] J. D. Bjorken, S. J. Brodsky and H. J. Lu, Phys. Lett. B286, 153 (1992); H. J. Lu, S. J. Brodsky and V. A. Khoze, Phys. Lett. B312 215 (1993).

[2] H. J. Lu, U. Md. preprint \#94-073, DOE/ER/40762-023 (1994), to be published in Nucl. Phys. B.

\section{Heavy Quark Physics}

\section{Model Independent Extraction of $V_{\mathrm{bc}}$ Without Heavy Quark Symmetry [1]}

In this report a new method to extract the CKM matrix element $\left|V_{b c}\right|$ was proposed based on a sum rule for semileptonic decays of the $B$ meson. The method relies on much weaker assumptions than previous approaches [2,3] which are based on heavy-quark symmetry, and indeed can be viewed as a generalization of them based only on the much weaker assumption that the virtual $c \bar{c}$ pair content of the $B$ meson can be neglected. In practice, the actual extraction of the CKM matrix element also requires the additional experimental condition that the sum rule saturates in the kinematically accessible region.

\section{(J. Milana and T. D. Cohen)}

[1] T. D. Cohen and J. Milana, Phys. Rev. D 50, R21 (1994).

[2] S. Nussinov and W. Wetzel, Phys. Rev. D 36, 130 (1987); M. B. Voloshin and M. A. Shifman, Sov. J. Nucl. Phys. 47, 511 (1988); N. Isgur and M. Wise, Phys. Lett. B232, 113 (1989); ibid, B237, 527 (1990).

[3] J. D. Bjorken, I. Dunietz and J. Taron, Nucl. Phys. B371, 111 (1992).

\section{QCD Sum Rules}

\section{The Electromagnetic Form Factor of the Pion and Instantons}

Since the beginnings of the QCD sum rule approach it was known that the underlying nonperturbative OPE seriously underestimates hadronic correlation functions with pseudoscalar 
currents. This can be attributed to the enhanced sensitivity of this channel to small-scale vacuum fields like instantons, which are left largely unaccounted for by the perturbatively calculated Wilson coefficients of the OPE. In accordance with this explanation, adding explicit instanton contributions to the OPE for the pseudoscalar correlation function was shown to stabilize the otherwise completely inconsistent QCD sum rule for the pion.

The electromagnetic form factor of the pion can also be calculated within the sum rule approach from the correlation function of two pseudoscalar interpolators and the electromagnetic current. Since this correlator will receive similarly dominant instanton contributions, it cannot be calculated reliably in the conventional OPE framework. Instead, we evaluate the leading, additional instanton contributions directly. Their study in the framework of the corresponding QCD sum rule is in progress.

The results will provide consistency checks both on an alternative QCD sum rule based on axial current interpolating fields, and on our approximations in the treatment of the instanton sector. Additionally, we hope to gain new and more detailed insight into generic aspects of instanton corrections to the OPE.

(H. Forkel, M. Nielsen and X. Jin)

Calculation of $\langle p|\bar{u} u-\bar{d} d| p\rangle$ from $Q C D$ Sum Rule and Estimate of the Neutron-Proton Mass Difference

We calculated the proton matrix element of the scalar-isovector current, $\langle p|\bar{u} u-\bar{d} d| p\rangle$, from the correlation function of proton interpolating field in an external scalar-isovector field using QCD sum rule method. The key phenomenological input is the response of $\langle 0|\bar{u} u-\bar{d} d| 0\rangle$ to the external field, which is estimated both from quark mass difference $\delta m=m_{d}-m_{u}$ and the isospin breaking in the quark condensates $\gamma=(\langle 0|\bar{d} d| 0\rangle-\langle 0|\bar{u} u| 0\rangle) /\langle 0|\bar{u} u| 0\rangle$ and from chiral perturbation theory.

We have shown that to the first order in the quark mass difference $\delta m$, the non-electromagnetic part of the neutron-proton mass difference can be estimated based on the quark mass difference $\delta m$ and the proton matrix element of the scalar-isovector current. The QCD sum rule prediction for the proton matrix element leads to an estimate of the neutron-proton mass difference which is in reasonable good agreement with the experimental value. The major uncertainty has been the uncertainty in the value of the response of $\langle 0|\bar{u} u-\bar{d} d| 0\rangle$ to the external field.

(X. Jin, M. Nielsen and J. Pasupathy (Indian Inst. of Science, Bangalore)) 


\section{Instanton Corrections to the Neutron-Proton Mass Difference}

We calculate the leading instanton corrections to the QCD sum rules [1] for the neutronproton mass difference $\delta M_{N}$. We discuss some particular aspects of instantons in the explicit flavor symmetry breaking sector and show that the instanton contributions resolve the significant discrepancies between the values of $\delta M_{N}$ found from the chirally even and odd sum rules.

The chirally even sum rule, which reproduced the phenomenological value of $\delta M_{N}$ already without instantons, remains unchanged (the leading instanton corrections to this sum rule are generally small and exactly zero for the standard "Ioffe current", which we use). The value of $\delta M_{N}$ from the chirally odd sum rule, however, which was originally found to be an order of magnitude too small, is increased to its phenomenological value by the instanton contributions, thus making the results from both sum rules consistent with each other and with experiment.

\section{(H. Forkel and M. Nielsen)}

[1] H. Forkel and M. K. Banerjee, Phys. Rev. Lett. 71, 484 (1993).

\section{QCD Sum Rules vs. Chiral Perturbation Theory}

QCD sum rules are useful tools for studying the spectral properties of hadrons; however, assumptions underlying standard sum rule analyses can lead to inconsistencies with known results of chiral perturbation theory. We have demonstrated this possibility in QCD sum rule extractions of the nucleon mass and $\sigma$ term.

QCD sum rules are based on the analytic properties of current-current correlators and asymptotic freedom. Hadronic spectral properties are related via dispersion relations to the current-current correlator evaluated in terms of fundamental quark and gluon fields. This evaluation uses an operator product expansion (OPE), which, in turn, requires knowledge of various vacuum matrix elements-the condensates. In practice, applications of QCD sum rules require simple parameterizations of spectral functions and a truncated OPE. These simplifications necessarily lead to uncertainties in the extracted spectral parameters; therefore, it is useful to test QCD sum rule results against reliable physical and mathematical constraints. We have used well-known properties of hadronic spectra in the chiral limit to test the dependability of QCD sum rule extractions of the nucleon mass and $\sigma$ term.

It is generally believed that chiral perturbation theory accurately describes all low-energy observables of QCD in the limit of light current quark masses. In the present context, however, we have only considered the leading nonanalytic behavior of observables as a function of $m_{q}$, the average of the up and down current quark masses. It should be noted that this 
nonanalytic behavior can be determined without the full machinery of chiral perturbation theory-it depends only on the existence of dispersion relations and a pseudo-Goldstone pion.

In principle, an exact evaluation of a current-current correlator in terms of QCD degrees of freedom must reproduce all of the hadronic physics, including the physics associated with the existence of a pseudo-Goldstone pion; therefore, an "exact" implementation of QCD sum rules must be consistent with chiral perturbation theory. As noted above, however, all practical implementations are not exact; thus practical applications of QCD sum rules are not necessarily consistent with chiral perturbation theory. By studying the magnitude of this inconsistency, we have estimated the scale of the uncertainties in QCD sum rule extractions of spectral parameters.

We have shown intrinsic uncertainties of $\sim 100 \mathrm{MeV}$ in QCD sum rule extractions of the nucleon mass and $\sigma$ term associated with inconsistencies between the sum rules and known chiral behavior. As a practical matter, this suggests that attempts to improve QCD sum rule calculations for the nucleon via an improved treatment of the OPE are of limited value in reducing the uncertainties. We find that a $100 \mathrm{MeV}$ uncertainty will persist until the inadequacies of the spectral ansatz are addressed.

(D. Griegel and T. D. Cohen)

\section{QCD Based Models}

\section{The Strange Vector Form Factors of the Nucleon [1]}

We have estimated the strangeness vector form factors of the nucleon in the momentum region relevant for the planned experiments at MIT-Bates and CEBAF, from our previously introduced model [2] of the nucleon strangeness distribution in terms of a kaon cloud, a vector meson dominance contribution and $\omega-\phi$ mixing. We found both Sachs form factors to be dominated by the VMD contribution. The electric strangeness form factor, in particular, is left practically unchanged if the intrinsic kaon cloud contribution is switched off. For the same reason the dependence of our result on the meson-baryon form factor masses in the kaon loops is very weak. Both the sign and the slope of the electric form factor are opposite to those of the pole-fit estimate. Together with the considerably larger slope of the pole-fit result at zero momentum transfer this leads already at moderate momentum transfer to a significant difference in magnitude between the two form factors.

Although the intrinsic kaon cloud contribution plays a somewhat more important role in the magnetic Sachs form factor, its overall shape and slope are again mainly determined by the vector mesons. The value at zero momentum transfer, i.e. the strangeness magnetic moment, however, does not receive VMD contributions and thus shows a more pronounced dependence on the intrinsic meson-baryon form factors. As in the case of the electric form 
factor, the overall qualitative behavior of our result, and in particular its slope and curvature, differs considerably from the pole fit estimate.

We further studied the nucleon strangeness radius from the point of view of a class of constituent quark models in which the constituent quarks have an extended structure. The valence up and down quarks in the nucleon can then acquire an intrinsic strangeness distribution. We pointed out that the strangeness radius of the nucleon is model-independently given by the sum of the constituent quark radii, since their strangeness charge distribution is isotropic and since their overall strangeness is zero. For a quantitative estimate we employed the Nambu-Jona-Lasinio model and found a 2-3 times smaller value than in the kaon-loopVMD model with the opposite sign.

We also reviewed and discussed some of the other existing theoretical estimates. Comparing the results for the strangeness radius and magnetic moment reveals in particular the large discrepancies between those predictions. Both signs of the strangeness radius, for example, and values within a range of an order of magnitude have been estimated. Clearly the theoretical uncertainties are at present not reliably under control and the existing results have to be regarded as order-of-magnitude estimates. The subject will remain intriguing and challenging in the years to come.

(H. Forkel, M. Nielsen, X. Jin and T. D. Cohen)

[1] H. Forkel, M. Nielsen, X. Jin and T. D. Cohen, U. Md. preprint \#94-097, DOE/ER/40762-030, submitted to Phys. Rev. D.

[2] T. D. Cohen, H. Forkel, and M. Nielsen, Phys. Lett. B316, 1 (1993).

\section{Nucleon Structure with Configuration Mixing}

Isgur, Karl and Koniuk [1] were the first to use configuration mixing in a quark model of the nucleon. Being based on a harmonic oscillator hamiltonian they had advantages, such as not having any center of mass motion at all, which we cannot have as we must mix configurations described with single quark spinors. Nevertheless we have tried to follow their approach as closely as possible.

We follow the following steps.

1. We begin by treating the Toy model at the mean field level. This yields a Dirac hamiltonian, $h$,

$$
h(\vec{r})=-i \vec{\alpha} \cdot \vec{\nabla}+\beta m_{q}+\beta \frac{g_{\pi} F_{\pi}}{\sqrt{\epsilon(\vec{r})}},
$$

where $\epsilon(\vec{r})$ is the color dielectric function inside the ground state of the nucleon as obtained in the mean field treatment of the Toy model. 
2. Next we generate the low-lying eigenspinors of the Dirac hamiltonian, $h$. We consider the 5 lowest single quark states which are: $1 s_{1 / 2}(342), 1 s_{1 / 2}(493), 1 p_{3 / 2}(553), 1 p_{1 / 2}(553)$, $1 d_{3 / 2}(718), 2 s_{1 / 2}(741)$, where the numbers in parantheses are the eigenvalues of the Dirac hamiltonian, $h$, in $M e V$.

3. One can construct altogether 10 states which are fully symmetric in space and isospin with $I=J=1 / 2$ and even parity. The configurations $\left(1 s \frac{1}{2}\right)^{3}$ and $\left(1 s \frac{1}{2}\right)^{2}\left(1 d \frac{3}{2}\right)$ produce one state each, while two distinct states are obtained from each of the following 4 configurations: $\left(1 s \frac{1}{2}\right)^{2}\left(2 s \frac{1}{2}\right),\left(1 s \frac{1}{2}\right)\left(1 p \frac{1}{2}\right)^{2},\left(1 s \frac{1}{2}\right)\left(1 p \frac{3}{2}\right)^{2}$, and $\left(1 s \frac{1}{2}\right)\left(1 p \frac{1}{2}\right)\left(1 p \frac{3}{2}\right)$.

4. Unlike the case of harmonic oscilator wave functions we cannot remove the motion of the center of mass in a simple manner. We try to minimize, as best as we can, the change in the center of mass motion, which is measured by the square of the momentum operator:

$$
\vec{P}=\int d^{3} x\left[-i \psi^{\dagger}(x) \vec{\nabla} \psi(x)+\dot{\chi}(x) \vec{\nabla} \chi(x)\right] .
$$

The contribution of the hybrid field, $\chi$, to $\vec{P}^{2}$ is $\sim 5 \%$ and is neglected in the present work.

5. The ground configuration expectation value of $\vec{P}^{2}$ is $\left\langle\left(1 s \frac{1}{2}\right)^{3}, I=J=1 / 2\left|\vec{P}^{2}\right|\right.$ $\left.\left(1 s \frac{1}{2}\right)^{3}, I=J=1 / 2\right\rangle=24.3 m_{\pi}^{2}$. We diagonalize $\vec{P}^{2}$ in the space of the 9 excited configurations and obtain the eigenvalues (in units of $m_{\pi}^{2}$ ): 28.0,31.2, 31.9, 32.1, 41.2, 41.6, 46.6, 47.8 and 57.9. We retain the 4 low eigenvalue states only and leave out the last 5 high eigenvalue states, thus restricting the spread of $\left\langle\vec{P}^{2}\right\rangle$ to $30 \%$ or less. Including the ground configuration, our configuration mixing calculation involves 5 linearly independent states.

6. We diagonalize the hamiltonian $H=H_{\text {Toy }}+H^{\prime}{ }_{t H o o f t}$. The strength of $H^{\prime}{ }_{t H o o f t}$ is adjusted to reproduce, after inclusion of the pionic contribution, the $N-\Delta$ mass splitting. The eigenvalues are corrected for the center of mass motion by subtracting the expectation value of $\vec{P}^{2} / 2 M_{0}$ in the corresponding eigenstate. Here $M_{0}=\left\langle N\left|H_{\text {Toy }}\right| N\right\rangle=$ $1400 \mathrm{MeV}$, where $N$ stands for the ground state of $H_{T o y}+H{ }_{t H o o f t}$.

7. Next we set up an effective hamiltonian for the $N, \Delta$ and $\pi$ system. Only states considered are $|N\rangle,|\Delta\rangle,|N, n \pi\rangle$ and $|\Delta, n \pi\rangle$, with $n=1,2$ only. The transition matrix elements, $\left\langle\Delta, \pi\left|H_{\text {int }}\right| N\right\rangle$ are constructed with the results of the Toy model with configuration mixing. The pionic effects are obtained perturbatively with this hadronic hamiltonian. Only those intermediate states which correspond to one loop are included in the calculations.

8. The resulting energies are listed in Table 1 . 
9. In Table 2 we list several nucleon properties obtained with and without $H_{t}{ }_{t H o o f t}$-mediated configuration mixing.

\begin{tabular}{|l|l|l|l|l|}
\hline 1 & 2 & 3 & 4 & 5 \\
\hline 984 & 1446 & 1529 & 1645 & 1913 \\
\hline
\end{tabular}

Table 1: The energies of the lowest five states of even parity and $I=J=1 / 2$. The effects of pion-quark interaction is included perturbatively, while the effects of the ' $t$ Hooft interaction is included by 5 selected configurations.

\begin{tabular}{|l|l|l|l|}
\hline Quantity & $\begin{array}{l}\text { Toy Model + } \\
\text { Pion Effect }\end{array}$ & $\begin{array}{l}\text { Toy Model + } \\
\text { Pion Effect }+ \\
\text { Config. mix } \\
\text { via 't Hooft }\end{array}$ & Exptal \\
\hline$M_{N}(\mathrm{MeV})$ & 1232 & 949 & 939 \\
\hline$M_{\Delta}(\mathrm{MeV})$ & 1231 & 1244 & 1232 \\
\hline$\left\langle r^{2}\right\rangle_{p}\left(\mathrm{fm}^{2}\right)$ & 0.76 & 0.64 & 0.66 \\
\hline$\left\langle r^{2}\right\rangle_{n}\left(\mathrm{fm}^{2}\right)$ & -0.09 & -0.13 & -0.12 \\
\hline p Mag. mom. & 2.55 & 2.27 & 2.79 \\
\hline n Mag. mom. & -1.83 & -1.52 & -1.91 \\
\hline$g_{\pi N N} \frac{m_{\pi}}{2 M_{N}}\left(m_{\pi}\right)$ & 0.99 & 0.78 & 1.00 \\
\hline
\end{tabular}

Table 2: Various properties of the nucleon with and without quark configuration mixing.

We consider the results quite satisfactory and hope that the situation with the magntic moments and $g_{\pi N N} \frac{m_{\pi}}{2 M_{N}}\left(m_{\pi}\right)$ can be improved with some readjustments of the parameters. This work is in progress.

(M. Kim and M. K. Banerjee)

[1] N. Isgur, G. Karl, and R. Koniuk, Phys. Rev. Lett., 41, 1269 (1978). 


\section{Relativistic Dynamics in Quark, Hadron and Nuclear Physics}

The most important new result in the last year is the proof of a low-energy theorem which establishes a condition for the validity of using the Dirac equation for composite, spin-1/2 particles. A low-energy theorem for scalar interactions has been proved which shows that the second-order interactions of a composite spin-1/2 system with an external scalar field are the same as those for a point Dirac particle interacting with a scalar potential. This theorem establishes a specific condition under which the z-graph contribution which is obtained by use of the Dirac equation for a composite system is valid. For reference, it is the z-graph contribution which has provided the interesting phenomenological successes for proton-nucleus scattering when the Dirac equation is used and which has been challenged as being doubtful due to compositeness of the nucleon. The key condition is that the scalar interaction must be such as to cause a mass shift and thus must be coupled to the divergence of the dilatation current of the system. We formulate this condition in very general terms. A field-theoretical model is developed for which explicit calculations give insight into the effect. The scattering from the constituents of the composite system cancels with off-shell contributions due to compositeness. There is no suppression of the composite system z-graph contribution when the condition is satisfied.

We also have considered the case of cancelling scalar and vector interactions because the scalar and vector interactions used in relativistic nuclear physics nearly cancel one another. The vector interaction considered does not obey a low-energy theorem guaranteeing that it will generate the same result as for a point Dirac particle. However, we find in a specific model of compositeness that scalar and vector interactions which cancel one another in first order again reproduce the result of the Dirac equation in second order. The z-graph contribution for the composite system is suppressed in this case due to the vector part of the interaction. However, off-shell and contact terms restore the result to that of the naive Dirac equation.

A workshop on Relativity in the Deuteron was held at CEBAF in September of 1993. The issue of z-graphs was raised at that meeting and a collaboration was formed between S. J. Wallace, J. A. Tjon and F. Gross to study the Compton scattering, for which a long-established low-energy theorem was known. The work of Wallace reported above is an outgrowth of those initial studies and a jointly authored paper is being written.

The collaboration of Wallace with N. Devine, a Ph.D. student now employed as a postdoctoral associate at CEBAF, continues with the objective of preparing papers on our analysis of elastic electron-deuteron scattering. Extensive calculations were performed using the two-body Dirac equation and meson-exchange interactions to determine the deuteron ground wave functions. Meson-exchange currents have been included in the analysis of electromagnetic form factors. Important progress has been made in deriving the electromagnetic current 
operator appropriate for use with the three-dimensional wave functions, based on a quasipotential reduction of the Mandelstam formalism for currents in the Bethe-Salpeter formalism. The resulting current operator satisfies an exact Ward-Takahashi identity. The analysis requires a new formulation which is based on having the bound state wave functions in the Breit frame. However, it is not possible to obtain these by boosting kinematically the rest frame wave functions. Instead, one must resolve the dynamical equations in the Breit frame, thereby accomplishing a dynamical boost. The formalism has many attractive features and we believe it to present one of the best options for analyses of electromagnetic scattering from relativistic bound states. Although the initial work is done for the specific case of the deuteron, the methods are generally useful for relativistic bound states.

In the past year, we have solved the boost problem in a simple case (separable interactions) and demonstrated the validity of our approach. Much more formidable is the case where the interactions are due to meson exchanges and we have restructured our efforts in order to work on that aspect. Ph.D. student P. Dulany and Wallace will undertake to solve the 'boost' problem for the general case. Meanwhile, a paper is being prepared (Devine and Wallace) to publish the results which have been obtained for electron-deuteron scattering.

C. E. Bell completed a Ph.D. thesis in the summer of 1993 which involved extensive calculations of the relativistic bound states of $\bar{q} q$ mesons using the two-body Dirac equation. This work has led to a good reproduction of all meson masses based on a few parameters. An interesting point is that the lowest energy $J^{P}=0^{-}$state tends to dive towards zero energy when the gluon exchange coupling constant, $\alpha_{S}$, is increased to about 0.34 . Correspondingly, the charge conjugate $0^{-}$state with negative energy ascends towards zero energy. The mass gap between the positive- and negative-energy states disappears at the critical coupling value, signalling the breakdown of the vacuum state into pairs of $0^{-}$mesons. The breakdown is similar to the mechanism of Cooper pairing in superconductors. It is possible to obtain a $135 \mathrm{MeV} \pi$-meson mass and an $800 \mathrm{MeV} \rho$-meson mass for a value of $\alpha_{S}$ near the critical value. At this point, Dr. Bell has left Maryland for a year of travelling. A paper has been drafted and we have decided to perform additional calculations to incorporate an interesting effect which is required to explain the Regge behavior of meson masses. The scalar confining interaction which agrees with the spin-dependence of QCD at long distance does not contain terms dependent on $L^{2}$ which arise from QCD and which have a beautiful explanation in terms of the rotational energy of a relativistic string, or flux tube. The extra rotational terms have been formulated and we are working to incorporate them into the calculations before submitting a paper, which now is in draft form.

An analysis of relativistic corrections in the three-body bound state has been underway for about two years. This work has progressed substantially due to the efforts of P. Dulany and we have recently extended the scope of the work. Our analysis is based on an instant 
quasipotential reduction in the rest frame of the three particles, which we have taken to be bosons for simplicity. The usual partial-wave analysis which applies for a nonrelativistic system fails for the relativistic case. Thus we have implemented a direct numerical solution of the three-body equations not using partial wave expansions. Solutions for the three-body ground state for three scalar particles (bosons) have been obtained using the CRAY computers at NERSC. We have evaluated the effects of differing forms of three-dimensional dynamics and a paper on the results should be finished shortly. In this work, the effects considered are mainly kinematical, although we expect that they provide the bulk of the relativistic correction.

The most interesting effect which arises in the instant formulation is the boost of the twobody interactions. This is quite nontrivial and it is an important unsolved problem in the field. We have formulated the 'boost' problem based on the same considerations which have been used in our electron-deuteron scattering work. The essential idea is to formulate the three-dimensional dynamics as a consistent quasipotential reduction of the underlying fourdimensional dynamics. This allows us to develop a 'boost' equation which specifies how the two-body interaction must change when it is evaluated at different values of the total threemomentum, such as in the c.m. frame of the two particles versus the rest frame of a three-body

system. P. Dulany is undertaking a theoretical analysis of the 'boost' problem relevant to the three-body bound state problem. Progress on this unsolved problem is essential to specifying a consistent three-dimensional reduction for analysis of relativistic bound states.

\section{A. Low-Energy Theorem for Scalar Interactions of a Composite Spin-1/2 System}

The use of the Dirac equation in nuclear physics has been a subject of interest and debate in recent years. One of the outstanding successes is in elastic scattering of protons by nuclei. Quite large scalar and vector interactions, which almost cancel one another, characterize the proton-nucleus interaction. Solving the Dirac equation with an attractive scalar potential and a repulsive vector one, each of magnitude about $300 \mathrm{MeV}$, produces a good description of spin observables at intermediate energies. The principal effect is due to z-graphs when the Dirac equation is used, but it may be understood also at a simpler, classical level.

Consider a classical hamiltonian of the form

$$
H=V+\sqrt{(M+S)^{2}+\mathbf{p}^{2}}
$$

where $V$ is the time-component of a vector potential and $S$ is a scalar potential, both of which are taken to be spatially uniform. Expanding in $S$ to second order yields,

$$
H=\epsilon+V+\frac{M}{\epsilon} S+\frac{\mathbf{p}^{2}}{2 \epsilon^{3}} S^{2}+\cdots
$$


where $\epsilon=\sqrt{M^{2}+\mathbf{p}^{2}}$. Consider the case of exact cancellation of the scalar and vector potentials: $V+\frac{M}{\epsilon} S=0$, for which,

$$
H=\epsilon+\frac{\mathbf{p}^{2}}{2 \epsilon^{3}} S^{2}+\cdots .
$$

The momentum dependent repulsive potential term in Eq. (3) provides the main relativistic effect in proton scattering by nuclei at intermediate energies.

To obtain the same results from the Dirac equation with scalar and vector potentials, one reduces the energy expression to the form,

$$
E \psi=\left(\epsilon+\mathcal{V}^{++}+\mathcal{V}_{p a i r}\right) \psi,
$$

where

$$
\mathcal{V}^{++}=V+\frac{M}{\epsilon} S
$$

and

$$
\nu_{\text {pair }}=\frac{\mathbf{p}^{2} S^{2}}{\epsilon^{2}[E+\epsilon-V+(M / \epsilon) S]}=\frac{\mathbf{p}^{2} S^{2}}{2 \epsilon^{3}}+\cdots,
$$

is the z-graph contribution. Correct to order $S^{2}$ it is the same as in Eq. (3).

For a composite system such as a nucleon, general arguments have been presented that z-graphs should be suppressed strongly. Thus the correctness of $\mathcal{V}_{\text {pair }}$ as obtained from the Dirac equation for a nucleon is in dispute, notwithstanding the classical basis for the effect.

A resolution of this apparent conflict is that there is a low-energy theorem for scalar interactions which guarantees the correctness of the $S^{2} \mathbf{p}^{2} /\left(2 \epsilon^{3}\right)$ term in Eq. (3) or Eq. (6). The result is quite general. It amounts to the observations 1.) that an external scalar interaction $S$ may be defined which acts to shift the mass of any composite system from $M$ to $M+S$, and 2.) that a composite spin- $1 / 2$ bound state with shifted mass $M+S$ has the energy expansion discussed above.

Analysis of a simple model demonstrates the origin of the expected result of Eq. (3) and clarifies the relative contributions of z-graphs, off-shell effects and contact interactions of a composite system. We consider the lagrangian,

$$
\mathcal{L}=\bar{\psi}(i \not \partial-m) \psi+\frac{1}{2}\left[\partial_{\nu} \phi \partial^{\nu} \phi-\mu^{2} \phi^{2}\right]+g \bar{\psi} \psi \phi^{2}
$$

Assume that this system has a bound state of mass $M$ and spin- $1 / 2$ which is composed of the elementary fermion of mass $\mathrm{m}$ and the scalar boson of mass $\mu$. By scaling all parameters in the lagrangian with dimensions of mass to new values $m \rightarrow \lambda M, \mu \rightarrow \lambda \mu, g \rightarrow \lambda^{-1} g$, and similarly scaling all cutoff or renormalization masses associated with the theory, it is clear that the bound state mass $M$ will be scaled to $\lambda M$. The desired result is obtained by choosing $\lambda=1+S / M$. The same procedure can be applied to any lagrangian with the same result, namely, $M \rightarrow M+S$. 
Expanding to first order in $S$, one finds that the lagrangian after mass scaling takes the form

$$
\mathcal{L} \rightarrow \mathcal{L}-\rho S,
$$

where the scale-breaking charge associated with the mass scaling is

$$
\rho=(m / M) \bar{\psi} \psi+2(\mu / M) \phi^{2}-(g / M) \bar{\psi} \psi \phi^{2} .
$$

In general, the scale-breaking charge $\rho$ which is associated with a mass scaling is proportional to the divergence of the dilatation current of the system, including anomalous contributions. For example, in the model of Eq. (7), a cutoff is required to obtain finite results. When a Pauli-Villars cutoff term involving a cutoff mass $\Lambda$ is added to $\mathcal{L}$, a corresponding anomalous contribution to $\rho$ is obtained by scaling the cutoff mass in the same way as the other parameters with dimensions of mass.

The propagator of the composite spin- $1 / 2$ system of mass $M$ and four-momentum $p$ may be written as,

$$
G(p)=\frac{1}{1-\Sigma(p)}
$$

where $\Sigma(p)$ takes the general form,

$$
\Sigma(p)=A\left(p^{2}\right) p+B\left(p^{2}\right)
$$

and $A$ and $B$ are scalar functions. Although a detailed expression for $\Sigma(p)$ is not required, it is straightforward to obtain one from the lagrangian of Eq. (7). In that case, $\Sigma$ is obtained from a loop graph involving a fermion and a scalar meson propagator.

By assumption, there is a pole in $G(p)$ at $\not p=M$. Expanding the propagator about the bound-state pole, one finds

$$
\begin{gathered}
G(p)=Z_{2}\left(\frac{1}{p-M}+G^{\prime}(p)\right), \\
Z_{2} \equiv-\left\{A_{0}+2 M^{2}\left[M A_{0}^{\prime}+B_{0}^{\prime}\right]\right\}^{-1},
\end{gathered}
$$

is a normalization factor with $A_{0}=A\left(M^{2}\right), A_{0}^{\prime} \equiv d A\left(p^{2}\right) /\left.d p^{2}\right|_{p^{2}=M^{2}}$, and similarly for $B_{0}^{\prime}$. Nonelementary propagation due to excited states of invariant masses greater than $M$ gives rise to

$$
G^{\prime}(p)=\frac{A_{0}^{\prime} p-B_{0}^{\prime}}{A_{0}}+\frac{D_{0}(p+M)}{A}+\cdots
$$

where terms omitted from the expansion are higher order in $p^{2}-M^{2}$ and do not play a role in the low-energy limit. Moreover, the detailed form for the function $D_{0}$ is not required.

The usual spectral expansion of the positive-energy pole term in $G(p)$ shows that the ground state of the composite system has the positive-energy wave function $Z_{2}^{1 / 2} u(p)$, where $u(p)$ is a Dirac spinor for an elementary fermion of mass $M$. 
Interactions of the composite system with an external vector field are described by a vertex function which, for zero momentum transfer, is given by

$$
\Lambda^{\mu}(p, p)=-\frac{\partial \Sigma(p)}{\partial p_{\mu}}=-A_{0} \gamma^{\mu}-2 p^{\mu}\left(A_{0}^{\prime} p+B_{0}^{\prime}\right)
$$

Making the very basic assumption that the scalar interaction is given by the scale-breaking charge $\rho$, we obtain the scalar vertex from Eq. (9) as follows,

$$
\Lambda^{\rho}(p, p)=\frac{m}{M} \frac{\partial \Sigma(p)}{\partial m}+\frac{\mu}{M} \frac{\partial \Sigma(p)}{\partial \mu}-\frac{g}{M} \frac{\partial \Sigma(p)}{\partial g}+\frac{\Lambda}{M} \frac{\partial \Sigma(p)}{\partial \Lambda}
$$

where $\Lambda$ is the cutoff mass. This can also be written as

$$
\Lambda^{\rho}(p, p)=-\frac{p_{\alpha}}{M} \frac{\partial \Sigma(p)}{\partial p_{\alpha}}=-A_{0} \frac{p}{M}-\frac{2 p^{2}}{M}\left(A_{0}^{\prime} p+B_{0}^{\prime}\right)
$$

Equation (16) is a direct consequence of a Ward identity for the divergence of the dilatation current. It may be derived from the fact that $\Sigma(p, m, \mu, g, \Lambda)$ is invariant when all parameters with the dimensions of mass are scaled, e.g.,

$$
\Sigma\left(m, \mu, \Lambda, g, p_{\alpha}\right)=\Sigma\left(\lambda m, \lambda \mu, \lambda \Lambda, \lambda^{-1} g, \lambda p_{\alpha}\right)
$$

Expanding to first order about $\lambda=1$, one finds the equality of Eqs. (15) and (16). The low-energy theorem which we discuss of course depends on the existence of such a Ward identity.

For comparison with the case of interest in nuclear physics, we study the scattering of a composite spin-1/2 system by a combination of static external scalar and vector fields which are cancelling: $V=-(M / \epsilon) S$. This corresponds to an interaction $S \Lambda^{S V}$, where $\Lambda^{S V}$ combines scalar and vector vertices as follows,

$$
\Lambda^{S V}(p, p) \equiv \Lambda^{\rho}(p, p)-(M / \epsilon) \Lambda^{0}(p, p)
$$

When $p^{2}=M^{2}$, one finds

$$
\Lambda^{S V}(p, p)=-A_{0}\left[(p / M)-(M / \epsilon) \gamma^{0}\right]
$$

Forward scattering of the composite fermion from the cancelling scalar and vector fields is studied in second order and in the limit $q \rightarrow 0$, where $q$ is the momentum exchanged with the source. After a straightforward analysis, we find the potential experienced by the composite system to be,

$$
\mathcal{V}=\frac{\mathbf{p}^{2} S^{2}}{2 \epsilon^{3}}\left[Z^{2}+\left(Z-Z^{2}\right)+(1-Z)\right]=\frac{\mathbf{p}^{2} S^{2}}{2 \epsilon^{3}}
$$

where $Z \equiv-Z_{2} A_{0}$ combines wave function and vertex factors. The cancellations which render the result independent of the factor $Z$ provide the low-energy theorem. They are quite general 
in origin. A scalar interaction coupling to mass plus a cancelling vector interaction generates a repulsive potential of the same form as that obtained classically from a mass shift in Eq. (1) or from the Dirac equation with scalar and vector potentials via $\mathcal{V}_{\text {pair }}$.

\section{(S. J. Wallace, F. L. Gross and J. A. Tjon)}

\section{B. Relativistic Analysis of the Deuteron and Elastic Electron-Deuteron Scatter- ing}

We have made significant progress [1] in the application of the quasipotential formalism to electromagnetic scattering from relativistic two-body bound states. Previously we solved for deuteron wave functions in the center-of-mass frame using the two-body Dirac equation developed by Mandelzweig and Wallace and a one-boson-exchange potential based on the Bonn potential. The wave functions have the quasipotential constraint of zero relative energy (corresponding to the two nucleons being equally off-shell). Impulse-approximation and meson-exchange-current contributions to elastic electron-deuteron form factors were calculated. However the approximations used to boost the wave functions were found to cause a small but significant ambiguity in the results. The basic problem is that the equally off-shell constraint, when applied to both initial and final deuteron wave functions, is inconsistent with conservation of four-momentum at the photon-nucleon vertex and with current conservation.

Last year we reported that the boost and current conservation problems were overcome by using the quasipotential constraint of zero relative energy in the Breit frame. For elastic e-d scattering, this single constraint can be applied to both initial and final two-body wave functions consistent with four-momentum conservation. The use of a single consistent constraint has made it possible to define a conserved current which is derived from a quasipotential five-point kernel by methods analogous to those used to obtain the two-body quasipotential kernel. Unlike previous work on this problem, our definition of the quasipotential five-point kernel is free of singularities. Analysis of the quasipotential five-point kernel yields an impulse approximation current operator based on five-point box and crossed box diagrams. This current operator obeys an exact Ward-Takahashi identity in all energy sectors $(++,+-,-+$, $--)$, thus ensuring current conservation in an appropriate way.

We implemented this formalism by calculating wave functions in the Breit frame with zero relative energy for each desired value of (non-zero) total momentum. Impulse approximation and meson-exchange-current form factors were calculated using wave functions with zero relative energy in the Breit frame.

A key point is to determine the quasipotential in the Breit frame where total threemomentum is $\mathbf{P}_{2}=-(1 / 2) \mathbf{q}$, assuming it is known in the c.m. frame where total three- 
momentum is $\mathbf{P}_{1}=0$. We have derived the following 'boost' equation,

$$
V_{P_{2}}=V_{P_{1}}+V_{P_{1}}\left[\Delta\left(P_{2}\right)-\Delta\left(P_{1}\right)\right] V_{P_{2}}
$$

where $\Delta(P)=G_{B S}(P)-G_{Q P}(P)$ is the difference of Bethe-Salpeter and quasipotential propagators. For the case of a separable interaction, this equation has been solved exactly and we have verified explicitly that use of the quasipotential at different momenta produces the same two-body binding. The problem is now being studied based on a meson-exchange interaction in collaboration with P. Dulany.

A long paper presenting the results for the deuteron form factors is in preparation.

(N. Devine and S. J. Wallace)

[1] N. K. Devine, Ph.D. thesis, University of Maryland, 1992.

\section{Quark-Antiquark Bound States Based on Two-Body Dirac Equation}

The Ph.D. thesis research of C. E. Bell was completed in the summer of 1993. A momentumspace analysis was carried out for relativistic bound states (mesons) formed of a quark and an antiquark. The analysis is based on the two-body Dirac equation derived from QED by Mandelzweig and Wallace, which takes the form,

$$
\left[\left(E_{1}-h_{1}\right) \frac{h_{2}}{\epsilon_{2}}+\left(E_{2}-h_{2}\right) \frac{h_{1}}{\epsilon_{1}}\right] \psi(\vec{p})=\int \frac{d^{3} p^{\prime}}{(2 \pi)^{3}} \hat{V}\left(\vec{p}-\overrightarrow{p^{\prime}}\right) \psi\left(\overrightarrow{p^{\prime}}\right) .
$$

where $\hat{V}=\gamma_{1}^{0} \gamma_{2}^{0} V$ and $h_{i}$ is the Dirac hamiltonian for particle $\mathrm{i}$.

The bound state spectrum is obtained for a potential consisting of linear confining and constant terms (scalar) plus a gluon exchange term (vector), as follows.

$$
V(r)=\kappa r+C+\frac{\alpha_{s}}{r} \gamma_{1} \cdot \gamma_{2}
$$

Analysis of the long-distance behavior of the interaction between two static quarks suggest that the potential grows linearly with distance. Moreover, the leading order in $m^{-1}$ corrections have the same spin dependence as is obtained from matrix elements of a Lorentz-scalar interaction. However, a number of calculations of meson spectra based on this have demonstrated that a scalar, linear potential used in a relativistic equation fails to give Regge behavior, $M^{2}=\alpha J$, where $\mathrm{J}$ is the angular momentum.

An alternative, which does yield improved Regge behavior, is to use a mixture of Lorentzscalar and Lorentz-vector terms. In order to gain some insight into this observation, consider 
the reduction in powers of $p / m$ of a scalar potential in positive-energy states. Expanding the Dirac matrix element of the interaction yields,

$$
\begin{array}{r}
V^{++,++}(\nabla, r)=V^{s c}(r)+\frac{1}{8}\left(\frac{1}{m_{1}^{2}}+\frac{1}{m_{1}^{2}}\right)\left(\nabla^{2} V^{s c}(r)+V^{s c}(r) \nabla^{2}\right) \\
-\frac{\sigma_{1} \cdot \nabla}{2 m_{1}} V^{s c}(r) \frac{\sigma_{1} \cdot \nabla}{2 m_{1}}-\frac{\sigma_{2} \cdot \nabla}{2 m_{2}} V^{s c}(r) \frac{\sigma_{2} \cdot \nabla}{2 m_{2}}
\end{array}
$$

where terms order $m^{-4}$ are omitted. The omitted terms are not negligible when quark masses are small and that is why the full momentum-space structure is solved in our calculations. For the case of a scalar confinement potential $\hat{V}^{s c}(r)=\kappa r$, the effective spin-independent potential in states of angular momentum $l$ is

$$
\begin{array}{r}
V_{e f f}^{s c}(r) \approx \kappa r-\frac{\kappa}{2}\left(\frac{1}{m_{1}^{2}}+\frac{1}{m_{2}^{2}}\right)\left(\frac{l(l+1)}{r}+\frac{1}{2 r}-r \nabla_{r}^{2}\right) \\
-\frac{\kappa}{2 r}\left(\frac{\mathbf{S}_{1} \cdot \mathbf{L}}{m_{1}^{2}}+\frac{\mathbf{S}_{2} \cdot \mathbf{L}}{m_{2}^{2}}\right)
\end{array}
$$

where $\mathbf{S}_{i}=\frac{1}{2} \sigma_{i}$. Note that the centrifugal terms proportional to $\kappa l(l+1) / r$ are attractive for the scalar case. This is consistent with the findings that masses do not increase appropriately with $J$ at high $J$ values.

In contrast, for the vector confinement potential, $\hat{V}_{v e c}=\kappa r$, we have

$$
\begin{aligned}
V_{e f f}^{v e c}(r) \approx \kappa r & +\frac{\kappa}{m_{1} m_{2}}\left(\frac{l(l+1)}{r}-\frac{1}{2 r}-\frac{d}{d r}-r \nabla_{r}^{2}+\frac{S_{1 r} S_{2 r}-3 \mathbf{S}_{1} \cdot \mathbf{S}_{2}}{r}\right) \\
& +\frac{\kappa}{4 r}\left(\frac{1}{4 m_{1}^{2}}+\frac{1}{4 m_{2}^{2}}\right)+\frac{\kappa}{r}\left(\frac{\mathbf{S}_{1} \cdot \mathbf{L}}{m_{1}^{2}}+\frac{\mathbf{S}_{2} \cdot \mathbf{L}}{m_{2}^{2}}+\frac{\left(\mathbf{S}_{1}+\mathbf{S}_{2}\right) \cdot \mathbf{L}}{m_{1} m_{2}}\right)
\end{aligned}
$$

The centrifugal terms proportional to $\kappa l(l+1) / r$ are repulsive for the vector case.

The long-distance potential deduced from the Wilson loop analysis of QCD has the following the expansion at low velocity,

$$
\begin{aligned}
V^{W L}(r)=\kappa r+\frac{\kappa}{36 r}\left(\frac{1}{m_{1}^{2}}+\frac{1}{m_{2}^{2}}+\right. & \left.\frac{8}{m_{1} m_{2}}\right)-\frac{\kappa}{6}\left(\frac{1}{m_{1}^{2}}+\frac{1}{m_{2}^{2}}-\frac{1}{m_{1} m_{2}}\right) \\
& \times \frac{l(l+1)}{r}-\frac{\kappa}{2 r}\left(\frac{\mathbf{S}_{1} \cdot \mathbf{L}}{m_{1}^{2}}+\frac{\mathbf{S}_{2} \cdot \mathbf{L}}{m_{2}^{2}}\right) .
\end{aligned}
$$

The spin-orbit terms agree with those in Eq. (25) based on a scalar confining potential. However, the centrifugal terms are much smaller than for a static scalar potential. For equal quark masses, they are one-sixth as large. Neither the Lorentz-scalar nor Lorentz-vector potentials, nor a linear combination of them, reproduces both the centrifugal and spin-orbit terms expected from the Wilson loop analysis QCD. 
Olson et al.have demonstrated that the centrifugal terms in the interaction at long distance agree with a low velocity expansion of a classical rotating string. Moreover, the string model is consistent with Regge behavior at high $\mathrm{J}$ and the spin dependence of the rotating string is argued to be similar to that of a scalar potential. Rotational energy of the string itself is the ingredient which leads to the same centrifugal terms as follow from the Wilson loop analysis of QCD, Eq. (27). Thus the long-distance properties of the QCD confining interaction between $q$ and $\bar{q}$ appear to be consistent with a Lorentz scalar, linear potential supplemented by rotational terms associated with the gluon fields that cause confinement. This qualitative picture is being explored in our work by inclusion of a rotational term in the scalar part of the potential as follows,

$$
V_{l}^{s c}(r)=C+\kappa r+\kappa^{\prime} \frac{l(l+1)}{r},
$$

where $\kappa^{\prime}$ is a parameter motivated by the rotational energy terms which are found in longdistance QCD or in a string model. Because this is the Lorentz scalar potential, when Dirac matrix elements are evaluated the $\kappa r$ term produces the terms in the effective potential of Eq. (25) and the $\kappa^{\prime}$ term should correspond to the difference between that and the expected behavior of Eq. (27). In practice, $\kappa^{\prime}$ is selected to provide a reasonable Regge slope for the $\pi-a_{1}$ masses.

Masses of the $\pi$ and $\rho$ mesons vary differently as functions of the coupling constant $\alpha$. For $\alpha=0$ there is no hyperfine splitting and the two masses coincide approximately. As $\alpha$ is increased the splitting between the $0^{--}$and the $1^{--}$increases. At a critical value of $\alpha$, $\alpha_{\text {critical }} \approx 0.34$, the pion mass goes to zero.

The phenomenon of diving has been exploited to obtain a light $(135 \mathrm{MeV}) \pi$ meson and a heavy $(800 \mathrm{MeV}) \rho$ meson. It is found that all known mesons can be described rather well with the two-body Dirac equation and the simple potential given above. Extensive calculations have been performed for the spectrum and wave functions of all mesons. However, the term proportional to $\kappa^{\prime}$ needs to be added to complete the work.

(C. E. Bell and S. J. Wallace)

\section{Quasipotential Approach to the Relativistic Three-Body Problem}

Quasipotential equations for three Dirac particles have been derived as a simple extension of the two-body equation for Dirac particles. (see S. J. Wallace, in Relativistic Methods in Nuclear Many-Body Physics, B. C. Clark, R. J. Perry and J. P. Vary eds. (World Scientific, Singapore, 1989), pp.395-409.). Partial wave analysis is quite cumbersome for such equations and thus it is of interest to develop a direct numerical solution. One issue which arises may be studied in a much simpler context, namely, three body bound states using a local potential 
omitting spin. In the simplest form of the problem, one has in the rest frame $p_{3}=-p_{1}+p_{2}$. The three-body equation is,

$$
\begin{aligned}
\psi\left(\vec{p}_{1}, \vec{p}_{2}, \vec{p}_{3}\right)= & G_{0}\left(\vec{p}_{1}, \vec{p}_{2}, \vec{p}_{3}\right) \int \frac{d^{3} q}{(2 \pi)^{3}} V(q)\left[\psi\left(\vec{p}_{1}-\vec{q}, \vec{p}_{2}+\vec{q}, \vec{p}_{3}\right)\right. \\
& \left.+\psi\left(\vec{p}_{1}-\vec{q}, \vec{p}_{2}, \vec{p}_{3}+\vec{q}\right)+\psi\left(\vec{p}_{1}, \vec{p}_{2}+\vec{q}, \vec{p}_{3}-\vec{q}\right)\right] .
\end{aligned}
$$

Direct numerical solutions of a form of this equation have been reported by Kalusch and Glockle (Z. Phys.) using the nonrelativistic propagator

$$
G_{0}\left(\vec{p}_{1}, \vec{p}_{2} \vec{p}_{3}\right)=\frac{1}{E-\left(p_{1}^{2}+p_{2}^{2}+p_{3}^{2}\right) /(2 M)}
$$

where $E$ is the binding energy $(E<0)$. The analysis of Kalusch and Glockle uses Jacobi coordinates such that one of the three integrations may be done analytically. To avoid any restriction to nonrelativistic kinematics, the full three-dimensional integration is done numerically in our work. Direct integration of the three-particle equation is known to involve singularities arising from the noncompact kernel. For some cases of interest, it is nevertheless possible to obtain a solution for the ground state of the system. We have developed a computer code which reproduces the results of Kalusch and Glockle, using CRAY time at NERSC to perform the calculations.

An interesting issue is the size of relativistic corrections to the three-body binding. We have addressed this issue by replacing the above nonrelativistic propagator by one involving relativistic kinetic energies as follows

$$
G_{0}\left(\vec{p}_{1}, \vec{p}_{2}, \vec{p}_{3}\right)=\frac{1}{3 M+E-\epsilon\left(p_{1}\right)-\epsilon\left(p_{2}\right)-\epsilon\left(p_{3}\right)}
$$

where $\epsilon(p)=\sqrt{p^{2}+M^{2}}$. This propagator is appropriate to the rest frame of the bound state in the relativistic quasipotential formalism when only positive-energy states are kept.

The relativistic two-body potentials are modified to the form $f(p) V\left(\mathbf{p}-\mathbf{p}^{\prime}\right) f\left(p^{\prime}\right)$, where $f(p)=m / \epsilon(p)$ or $f(p)=[m / \epsilon(p)]^{1 / 2}$, depending on the form of dynamics used. Calculations have been performed for both possibilities. We have solved the two-body problem and fitted the parameters of the potentials to the phase shifts obtained for the Malfliet-Tjon potential with nonrelativistic kinematics. The three-body bound states have been calculated with the refitted potentials and two versions of relativistic kinematics, one which follows from our twobody equation with neglect of negative-energy states and the other which has been derived by Coester and Glockle based on a hamiltonian formulation. In the relativistic case, the propagator cannot be diagonalized by use of the Jacobi momenta. We have solved the problem directly using gaussian integration, a spline interpolation algorithm and an iterative solution 
for the bound state, following the work of Kalusch and Glockle. Final results for the threebody binding are expected soon and a draft of a paper has been prepared.

In addition to the kinematical factors $f(p)$, it is necessary to modify the two-body potentials for different total three-momenta of the interacting pair. This modification is governed by Eq. (21) of subsection B. We are researching means to solve the 'boost' problem.

(A. Delfino, P. Dulany and S. J. Wallace) 


\section{Heavy Ion Dynamics and Sharp Lepton Pairs}

During the last year we have pursued research in two major areas of theoretical physics related to nuclear heavy ions processes, as summarized in the following.

\section{A. The Composite $\left(Q_{0}\right.$ ?) Particle Scenario and the Generalized "Sharp Lepton" Problem}

1. Recent Expansion of " $\left(e^{+} e^{-}\right)$-Puzzle" to the "Sharp Lepton Problem"

(a) Sharp Electrons from $\left(\beta^{+}+\right.$Atom) Expand the Range of Relevant Data

During the last few years, new studies of $\beta^{+}$irradiation of high-Z atoms (Sakai, et al.). have been published which tend to clarify previously contradicting data and to support the earlier evidence that sharp $\left(e^{+} e^{-}\right)$pairs are in fact produced in such processes. We have shown that these data may also be encompassed by the same Composite Particle Scenario, as has been applied to the " $\left(e^{+} e^{-}\right)$-Puzzle" of the high-Z heavy ion collisions. The sharp leptons here arise from the inverse process to the Sharp Annihilative Positron Emission (SAPosE) from the $\left(C\left(Q_{0}\right.\right.$ ?)-Atom) supercomposite molecule.

This SAPosE process had earlier been shown to predict sharp single-positron lines with energies in the range $620<E_{+}<950 \mathrm{keV}$ from heavy ion collisions (such as $U+T a$ ), in which the composite $C\left(Q_{0}\right.$ ?) particle emerges bound to a heavy ion (where the binding is indicated by a Coulomb shifted energy difference, $\left(E_{+}-E_{-}\right)$, distribution.) Unfortunately the positron detectors of the Argonne Lab's new APEX experiment will, like its predecessors, be unable to measure such energetic positrons.

If the sharp SAPosE positrons could be observed, then the inverse would necessarily be implied, in which an incident $\beta^{+}$particle of the correct resonant energy collides with a bound electron of the neutral target atom, and creates the Composite $C\left(Q_{0}\right.$ ? $)$ particle bound in a supercomposite molecular state with the atom. Subsequently, each eigenstate of the Composite particle may decay to a sharp $\left(e^{+} e^{-}\right)$pair. In fact, as outlined below, it apears that the inverse process may already be under study in the Sakai experiments.

\section{(b) Sakai's Sharp Electrons Stringently Constrain the Originating Decay Process}

We note that the above scenario does not immediately imply the very sharp $e^{-}$energies $(\Gamma \lesssim 1 \mathrm{keV})$ observed by Sakai. The reason is that such narrow widths imply a source which is nearly at rest $\left(v<10^{-3} c\right)$. But this source must also be isolated and NOT bound to a nuclear ion, else the lepton distribution is again Coulomb broadened. Therefore, one must assume that the supercomposite molecular state is slightly unbound in the case of neutral atom targets, 
that it breaks up before the $C\left(Q_{0}\right.$ ?) decays, and that the $C\left(Q_{0}\right.$ ?) emerges from the breakup process essentially at rest. The last requirement is novel and of special interest. It implies a process of "Viscous Breakup" for the supercomposite molecule during which the kinetic energy of the relatively rigid composite $C\left(Q_{0}\right.$ ?) particle is transferred entirely to the electron cloud of the atom. It thus requires the consideration of the electron- $C\left(Q_{0}\right.$ ?) interactions inside the atomic electron cloud. This interpretation also suggests new studies with Bhabha $e^{+}$beams impinging upon high- $\mathrm{Z}$ neutral atoms (which alone can bind, or almost bind, the $C\left(Q_{0}\right.$ ?) particle) as opposed to the low- $Z$ targets favored heretofore (because their bound electrons have a narrower momentum speed) in Bhabha studies.

\section{(c) Upper Bound on Break-up Lifetime of Supercomposite Molecule}

Sakai's $\beta^{+}$data also implies the first empirical upper bound upon the lifetime of the supercomposite state against breakup, $\tau_{M A X} \approx 10^{-17} \mathrm{sec}$, which becomes an additional characteristic of the $C\left(Q_{0}\right.$ ?) system, to be added to several other properties (mentioned below) previously suggested by the scenario.

\section{(d) Data of $\left(e^{+} e^{-}\right)$-Puzzle and Sharp $\beta^{+}$Leptons Combine Into Single Expanded} "Sharp Lepton Problem"

Thus the "Composite Particle (Quadronium?) Scenario" continues to expand its range of conceptual relevance, encompassing now the broader "Sharp Lepton Problem" which includes the data from $\beta^{+}$irradiations, as well as the heavy ion $\left(e^{+} e^{-}\right)$-Puzzle data. [J. J. Griffin in Topics in Atomic and Nuclear Collisions, ed. by B. Remaud et al. (Plenum Press, 1994), p. 419.]

(J. J. Griffin)

2. Previous Successes of the Composite Particle Scenario for Heavy Ion $\left(e^{+} e^{-}\right)$Puzzle

The scenario successfully describes the properties of the sharp lepton pairs observed in the EPOS-GSI collaboration in terms of the eigenvalues of the $C\left(Q_{0}\right.$ ?) particle and the LorentzDoppler shifts due to the motion of the $C\left(Q_{0}\right.$ ?) particle in the laboratory frame. From the non-zero average difference energies, $\Delta=E_{+}-E_{-}$, observed in the U+Ta collision, it suggests the existence of a new kind of "Supercomposite" molecular state in which the neutral polarizable composite $C\left(Q_{0}\right.$ ?) particle is bound to nuclear ion. Moreover, four new, so far unobserved, decay modes then become allowed to $C\left(Q_{0}\right.$ ?) which are forbidden by energy momentum conservation for free $C\left(Q_{0}\right.$ ?) decay. One, Sharp Annihilative Positron Emission (SAPosE), offers, as mentioned above, in its inverse process of Recoilless Positron 
Absorption (RePosA) the key to understanding the very sharp lepton lines observed to follow $\beta^{+}$irradiation of heavy atoms. These lines then become just another aspect of the $C\left(Q_{0}\right.$ ? $)$ scenario, generalizing " $\left(e^{+} e^{-}\right)$Puzzle" into the extended "Sharp Lepton Problem".

The $C\left(Q_{0}\right.$ ?) Scenario also implies a $C\left(Q_{0}\right.$ ?) REST ENERGY, $M_{C} c^{2} \sim 3 m c^{2}(\sim 1.5 \mathrm{MeV})$, and LEVEL SPACING, $D_{C} \leqslant 100 \mathrm{keV}$, both from the sharp $\left(e^{+} e^{-}\right)$lines' energies; and (for a $Q_{0}$ composite of rest energy, $M_{Q} c^{2} \approx 3 m c^{2}(\approx 1.5 \mathrm{MeV})$ with binding energy, $B_{Q} \sim m c^{2}$ $(\sim 0.5 \mathrm{MeV}))$ : a RADIUS roughly equal to the particle's own Compton wavelength, $R_{Q} \sim$ $\lambda_{c}^{Q} \sim \hbar / 3 \mathrm{mc}(\sim 135 \mathrm{Fm})$, and a monopole STIFFNESS, $k_{Q} \sim M_{Q} / R_{Q}^{2}\left(\sim 10^{-4} \mathrm{MeV} / \mathrm{Fm}^{2}\right)$, both from model polarization studies of the heavy ion creation process. [Int. J. Mod. Phys. $\underline{\text { A6, }}, 1985$ (1991); Phys. Rev. Lett. 66, 1426 (1991); 68, 1960 (1992); Topics in Atomic and Nuclear Collisions, ed. B. Remaud (Plenum Press, New York, 1994), p. 419.]

(J. J. Griffin)

3. New Interest in Bhabha Scattering from Heavy Atoms; No Constraints on Particle Lifetime from Bhabha Data on Light Atom Targets

Our previous analyses have shown that the lifetime constraints asserted from data on Bhabha scattering from light atoms, which seemed an obstacle to the Composite Particle Scenario, are in fact not empirical results at all, but are instead artifacts of the theoretical assumptions of the analysis: present Bhabha data can place no empirical constraints whatsoever upon the composite particle lifetime. [Phys. Rev. C C47, 351 (1993); Phys. Rev. Lett. $\underline{70}, 4158(1993)$.]

On the other hand, the extended scenario implies that Bhabha scattering from heavy atoms $(Z \sim 92)$ ought also to produce the sharp lines aboserved by Sakai et al. It therefore recommends a shift in the experimental attention away from light atoms, which minimize the momentum spread of the bound atomic target electrons towards heavy atoms, when the $C\left(Q_{0}\right)$ can be created in a non-recoilling supercomposite bound state, subsequently to escape and decay nearly at rest.

(J. J. Griffin)

4. How Does $\left(Q_{0}\right.$ ?) Impinge Upon High Precision Quantum Electrodynamics?

Neither Quantum Electrodynamics, nor any other branch of modern theoretical physics so far predicts a charge neutral particle of mass $\sim 1.5 \mathrm{MeV}$ and eigenvalue spacing $\sim 150 \mathrm{keV}$. Nevertheless, the $\left(e^{+} e^{-}\right)$data indicate that high- $Z$ heavy ion collisions create such a particle which subsequently decays into $\left(e^{+} e^{-}\right)$, but indicate also that it cannot be an elementary 
particle, nor (because of the small level spacing) a two-body bound state. The Composite Particle Scenario is therefore the simplest allowed by the data.

The question whether this particle is made primarily of leptons, as the Quadronium hypothesis, $C=Q_{0}=\left(e^{+} e^{+} e^{-} e^{-}\right)$, would assume is, however, not yet addressed by any data: any $C$-particle which decays to $\left(e^{+} e^{-}\right)$could describe the Doppler-Lorentz features of the EPOS data as well as $Q_{0}$. We have begun to address this problem by considering the implications for Quantum Electrodynamics from the existence of a bound composite particle of the $Q_{0}=e^{+} e^{+} e^{-} e^{-}$composition.

We find that the effect of the $Q_{0}$ on the theoretical value of $\left(g_{e}-2\right)$ has a magnitude comparable to the present theoretical error, and therefore offers, at best, only a prospective future test. More promising is the correction implied by QED for the $3 \gamma$ decay of Parapositronium, where an apparent discrepancy between theory and calculation (but one not yet firmly established due to the error of the present theoretical value) could arise from the additional corrections which the bound $Q_{0}$ would require.

In the search for phenomenologically relevant data, we are currently considering other ways in which Quantum Electrodynamics might be affected by a composite particle of the Quadronium $\left(e^{+} e^{+} e^{-} e^{-}\right)$type, and in particular whether QED as we know it can exclude such a particle. At present our work shows only that the question is still open, despite some assertions to the contrary, which under scrutiny turn out to apply only to very selected source particles.

(V. Hushevater and J. J. Griffin)

\section{Further Study of "Sharp Lepton Problem"}

Studies of various phenomenological aspects of this hypothetical Composite particle will continue during the next year. The work has two main goals: (a) to identify experimental data which speaks (or which will speak, when they become available) to the existence of such a particle and to its internal structure, and (b) of continuing to seek some plausible mechanism within conventional quantum electrodynamics by which such a particle could be self-bound. So far the continued expansion of our comprehension of the data at the phenomenological level has been the more rewarding line of inquiry.

(J. J. Griffin)

\section{B. Permeation Current and Observed $\bar{N}, \bar{Z}$ Drifts in Heavy Ion Collisions}

We have almost completed for publication the manuscript describing our study of the effects of the permeation current upon the observed $\bar{N}, \bar{Z}$ drifts in deep inelastic collisions. 
This report exhibits the essential role of the Quantal Permeation Current in providing an adequate statistical description for such drifts. (This report has been much delayed by the shifting of the commitments of each of the three participants to other areas of activity.)

(T. Kiess, M. Dworzecka and J. J. Griffin) 


\section{Research Publications: 1993-(July) 1994}

Published Papers: 1993

1. CHIRAL CORRECTIONS TO LATTICE CALCULATIONS OF CHARGE RADII, D. B. Leinweber and T. D. Cohen, Phys. Rev. D $\underline{47}, 2147$ (1993).

2. RESPONSE OF NUCLEON TO EXTERNAL PROBES IN HEDGEHOG MODELS: I. ELECTROMAGNETIC POLARIZABILITIES, W. Broniowski and T. D. Cohen, Phys. Rev. D $\underline{47}, 299$ (1993)

3. RESPONSE OF NUCLEON TO EXTERNAL PROBES IN HEDGEHOG MODELS: II. GENERAL FORMALISM, W. Broniowski and T. D. Cohen, Phys. Rev. D $\underline{47}, 1$ (1993).

4. LIFETIME OF $\left(e^{+} e^{-}\right)$PUZZLE'S COMPOSITE PARTICLE: NO VALID LIMITS YET, J. J. Griffin, Phys. Rev. C 뜨, 351 (1993).

5. FINITE TEMPERATURE QCD SUM RULES RE-EXAMINED $\rho, \omega, A_{1}$ MESONS, T. Hatsuda, Y. Koike, and Su H. Lee, Nucl. Phys. B $\underline{394}, 221$ (1993).

6. MODEL INDEPENDENCE OF SCATTERING OF THREE IDENTICAL BOSONS IN TWO DIEMENSIONS, S. K. Adhikari, A. Delfino, T. Frederico and L. Tomio, Phys. Rev. A 47,1093 (1993).

7. DO QUARKS REALLY FORM DIQUARK CLUSTERS IN THE NUCLEON?, D. B. Leinweber, Phys. Rev. D $\underline{47}, 5096$ (1993).

8. PATTERN OF CHIRAL RESTORATION AT LOW TEMPERATURE FROM QCD SUM RULES, T. Hatsuda, Y. Koike and Su H. Lee, Phys. Rev. D $\underline{47}, 1225$ (1993).

9. THE PION CLOUD IN QUENCHED QCD, T. D. Cohen and D. B. Leinweber, Com. on Nucl. and Part. Phys. 21, 137 (1993) [Gordon \& Breach, Singapore, 1993].

10. COMMENT ON 'SEARCH IN s-CHANNEL FOR PRODUCTION OF 1-2 $\mathrm{MeV} / \mathrm{c}^{2}$ LONG-LIVED $\left(e^{+} e^{-}\right)$RESONANCES' AND ON 'SEARCH FOR LOW MASS STATES IN ELASTIC $\left(e^{+} e^{-}\right)$SCATTERING', J. J. Griffin, Phys. Rev. Lett. $\underline{70}, 4158$ (1993).

11. DETERMINING THE PION FORM FACTOR AT HIGH $Q^{2}$, R. Kahler and J. Milana, Phys. Rev. D $\underline{47}$, R3690 (1993).

12. A ChIRAl CONFINING MOdel of THE NUCLEON, M. K. Banerjee, Prog. in Particle and Nuclear Physics, Vol. $\underline{31}$, A. Faessler, ed. (Pergamon Press, Oxford, 1993), pg. 77. 
13. EFFECTIVE INTERACTION FOR INELASTIC PROTON SCATTERING BASED ON THE RELATIVISTIC IMPULSE APPROXIMATION, R. J. Furnstahl and S. J. Wallace, Phys. Rev. C 4 7, 2812 (1993).

14. COULOMB EFFECT IN THE TRINUCLEON SYSTEM IN AN OPTICAL POTENTIAL MODEL, A. Delfino, published in Int. J. Mod. Phys. A (1993).

15. POWER FALLOFF OF PION FORM FACTOR, L. S. Kisslinger and S. W. Wang, published in Nucl. Phys. B (1993).

16. QCD SUM RULES FOR NUCLEONS IN NUCLEAR MATTER II, X. Jin, T. D. Cohen, R. J. Furnstahl and D. K. Griegel, Phys. Rev. C $\underline{47}, 2882$ (1993).

17. STRUCTURE OF'THE PION AND EFFECTIVE ELECTROWEAK CURRENTS IN SOLITON MODELS OF THE NUCLEON, W. Broniowski and T. D. Cohen, Phys. Rev. D $\underline{48}, 2299$ (1993).

18. RELATING PHYSICAL OBSERVABLES IN QCD USING THE EXTENDED RENORMALIZATION GROUP METHOD, H. J. Lu and S. J. Brodsky, Phys. Rev. D $\underline{48}, 3310$ (1993).

19. QED-INDUCED RAPIDITY-GAP EVENTS AT THE Z PEAK, H. J. Lu, S. J. Brodsky and V. A. Khoze, Phys. Lett. B $\underline{312}, 215$ (1993).

20. UNUSUAL, LARGE RAPIDITY GAP, JET EVENTS AT HERA: A PQCD APPROACH, H. J. Lu and J. Milana, Phys. Lett. B313, 234 (1993) .

21. NUCLEON SIGMA TERM FROM QCD SUM RULE, X. Jin, M. Nielsen and J. Pasupathy, Phys. Lett. B314, 163 (1993).

22. DIRECT INSTANTONS IN QCD NUCLEON SUM RULES, H. Forkel and M. K. Banerjee, Phys. Rev. Lett. $\underline{71}, 484$ (1993).

23. ROLE OF INSTANTONS IN A CHIRAL CONFINING MODEL, M. Kim and M. K. Banerjee, Phys. Rev. C $\underline{48}, 2035$ (1993).

24. BARYON OCTET TO DECUPLET ELECTROMAGNETIC TRANSITIONS, D. B. Leinweber, T. Draper and R. M. Woloshyn, Phys. Rev. D $\underline{48}, 2230$ (1993).

25. JUST HOW STRANGE? LOOPS, POLES AND THE STRANGENESS RADIUS OF THE NUCLEON, T. D. Cohen, H. Forkel, and M. Nielsen, Phys. Lett. B $\underline{316}, 1$ (1993). 
26. DOES $J / \psi \rightarrow \pi^{+} \pi^{-}$FIX THE ELECTROMAGNETIC FORM FACTOR $F_{\pi}(t)$ AT $t=M_{J / \psi}^{2}$ ?, J. Milana, S. Nussinov, and M. G. Olsson, Phys. Rev. Lett. 71, 2533 (1993).

27. HEAVY QUARK SYMMETRY AND PAIR PRODUCTION OF HEAVY MESONS: A PERTURBATIVE QCD ESTIMATE, T. D. Cohen and J. Milana, Phys. Lett. B306, 134 (1993).

28. ELECTROMAGNETIC SCATTERING FROM RELATIVISTIC BOUND STATES, N. K. Devine and S. J. Wallace, Phys. Rev. C $\underline{48}$, R973 (1993).

\section{Published Papers: 1994}

1. UNQUENCHING THE RHO MESON, D. B. Leinweber and T. D. Cohen, Phys. Rev. D $\underline{49}, 3512$ (1994).

2. QCD SUM RULES FOR NUCLEONS IN NUCLEAR MATTER III, X. Jin, M. Nielsen, T. D. Cohen, R. J. Furnstahl and D. K. Griegel, Phys. Rev. C $\underline{49}, 464$ (1994).

3. BUILDING A CHIRAL QUARK MODEL, M. K. Banerjee (review article), to appear in M. N. Saha Centenary issue of Indian J. of Phys. (1994).

4. COMPARISON BETWEEN RELATIVISTIC AND NONRELATIVISTIC MODELS OF THE NUCLEON-NUCLEON EFFECTIVE INTERACTION. I. NORMAL PARITY ISOSCALAR TRANSITIONS, J. J. Kelly and S. J. Wallace, Phys. Rev. C. $\underline{49}$, 1315 (1994).

5. ON THE A DEPENDENCE OF $R=\sigma_{L} / \sigma_{T}$ and the $Q^{2}$ DEPENDENCE OF SHADOWING, J. Milana, Phys. Rev. C $\underline{49}, 2820$ (1994).

6. GOLDSTONE PION AND OTHER MESONS USING A SCALAR CONFINING INTERACTION, F. Gross and J. Milana, to be published in Phys. Rev. D (1994).

7. DISORIENTED CHIRAL CONDENSATES AND QUANTUM MECHANICAL ISOSPIN CORRELATION, T. D. Cohen, M. K. Banerjee, M. Nielsen and X. Jin, to be published in Phys. Lett. B (1994).

8. QCD SUM RULES VS. CHIRAL PERTURBATION THEORY, D. K. Griegel and T. D. Cohen, to be published in Phys. Lett. B (1994)

9. QCD SUM RULES FOR $\Lambda$ HYPERON IN NUCLEAR MATTER, X. Jin and R. J. Furnstahl, Phys. Rev. $\underline{\mathrm{C} 49}, 1190$ (1994). 
10. THE SHARP LEPTON PROBLEM AND THE $C\left(Q_{0}\right.$ ?) SCENARIO, J. J. Griffin, Lectures given at the Predeal Int. Summer School in Physics, Predeal, Romania, 1992; in Topics in Atomic and Nuclear Collisions, (Plenum Pub., London, 1994).

11. RELATIVISTIC QUASIPOTENTIAL APPROACHES IN ELECTROMAGNETIC FORM FACTORS OF THE DEUTERON, S. J. Wallace and N. K. Devine, in Few-Body Problems in Physics '93, eds. B. L. G. Bakker and R. van Dantzig (Springer-Verlag, New York, 1994), pp. 395-408.

12. SYSTEMATICS OF EXCLUSIVE $\beta$-DECAYS: A PQCD Analysis, C. E. Carlson and J. Milana, U. Md. Phys. Rev. D $\underline{49}, 5908$ (1994).

13. MODEL-INDEPENDENT EXTRACTION OF $\left|V_{b c}\right|$ WITHOUT HEAVY QUARK, T. D. Cohen and J. Milana, Phys. Rev. D $\underline{50}$, R21 (1994).

14. RAPIDITY GAP IN JET EVENTS AT LEP200, H. J. Lu, Nucl. Phys. B (1994), to appear.

Invited Papers: 1993-94

1. MESON FORM FACTORS AT LARGE $Q^{2}$, J. Milana, Elba Conference on Exclusive Reactions at High Momentum Tansfers, Elba, Italy, 1993, eds. C. E. Carlson and P. Stoler (World Scientific, Singapore, 1994), to be published.

2. QUASIPOTENTIALS, QUASICURRENTS, QUASIBOOSTS AND ALL THAT, S. J. Wallace, CEBAF/INT Workshop on Relativistic Effects in the Deuteron, Newport News, VA, 1993.

3. RELATIVISTIC DESCRIPTION OF THE DEUTERON AND ITS ELECTROMAGNETIC INTERACTIONS, S. J. Wallace, Gordon Conf. on Simple Systems in Physics and Chemistry, Titlton, NH, 1993.

4. BARYONS IN NUCLEAR MATTER: A QCD SUM RULE APPROACH, T. D. Cohen, Gordon Conference, Tilton, NH, 1993.

5. COMPLEMENTARITY OF $\pi N, K N$ and $\gamma$ N REACTIONS IN BARYON SPECTROSCOPY, T. D. Cohen, Workshop on Future Directions in Particle and Nuclear Physics at MultiGeV Hadron Beam Facilities, Brookhaven National Laboratory, NY, 1993.

6. THE STRANGENESS RADIUS OF THE NUCLEON, T. D. Cohen, Int'l. Workshop on the Quark Structure of Nucleon, Trento, Italy, 1993. 
7. MODELS OF THE NUCLEON, M. K. Banerjee, Proc. of the International Conference on Astrophysics and Cosmology: ICAC '93, Calcutta, India (1993).

8. AN HOMAGE TO PROFESSOR MEGHNAD SAHA, M. K. Banerjee, to appear in Professor M. N. Saha Birth Centenary Commemorative Volume of Indian J. of Physics, ed. S. B. Karmohapatro (1994).

9. LEVINSON'S THEOREM FOR THE DIRAC EQUATION, M. K. Banerjee, to appear in Professor M. N. Saha Birth Centenary Commemorative Volume Indian J. of Physics, ed. S. B. Karmohapatro (1994).

10. SHARP $\left(e^{+} e^{-}\right)$PAIRS FROM $\left(\beta^{+}+\right.$ATOM $)$AND FROM HEAVY ION COLLISIONS, J. J. Griffin, Proc. of 10th Winter Workshop on Nuclear Dynamics, Snowbird, UT (1994), ed. W. Bauer (World Scientific, 1994).

\section{Contributed Papers: 1993-94}

(Published in Proceedings or Books)

1. QCD SUM RULES FOR HYPERONS IN NUCLEAR MATTER, X. Jin, R. J. Furnstahl and M. Nielsen, Proc. of HYP94 International Conference on Hypernuclear and Strange Particle Physics, TRIUMF, Vancouver, 1994 (to be published in Nuclear Physics A).

2. LARGE RAPIDITY GAP, JET EVENTS AT HERA: A PQCD APPROACH, J. Milana, Proc. of the $V^{\text {th }}$ Blois Workshop on Elastic and Diffractive Scattering, Providence, RI, eds. H. M. Fried, K. Kang and C.-I. Tan (World Scientific, Singapore, 1994), to be published.

3. ON THE A-DEPENDENCE OF $\sigma_{L} / \sigma_{T}$, J. Milana, Proc. of Workshop on CEBAF at Higher Energies, eds. C. E. Carlson and P. Stoler (World Scientific, Singapore, 1994), to be published.

4. DECAYS OF HEAVY MESON BOUND STATES, C. E. Carlson and J. Milana, Proc. $14^{\text {th }}$ Int 7 . IUCAP Conf. on Few Body Problems in Physics, Williamsburg, VA, 1994, ed. F. Gross, to be published.

5. THE $C\left(Q_{0}\right.$ ?) SCENARIO: BOUND SHARP POSITRON DECAY, ITS RESONANT INVERSE, AND SHARP LINES FROM $\left[\beta^{+}+\right.$ATOM], J. J. Griffin, Proc. of the Fifth Int'l. Conf. on Nucleus-nucleus Collisions, Taormina, Italy, 1994, eds. M. diToro and E. Migneco, Special Issue of Nucl. Phys. A, to be published. 


\section{Papers Submitted for Publication}

1. ON THE SELF-CONSISTENCY OF SCALE SETTING METHODS, S. J. Brodsky and H. J. Lu, U. of Md. PP\#93-087, DOE/ER/40322-186 (1992), submitted to Phys. Lett. B.

2. CHIRAL QUARK DYNAMICS IN DENSE NUCLEAR MATTER, H. Forkel, U. Md. PP\#94-037, DOE/ER/40762-017, submitted to Nucl. Phys. B.

3. CALCULATION OF $<p|\bar{u} u-\bar{d} d| p>$ FROM QCD SUM RULE AND THE NEUTRONPROTON MASS DIFFERENCE, X. Jin, M. Nielsen and J. Pasupathy, U. Md. PP\#94082, DOE/ER/40762-027, submitted to Phys. Rev. D.

4. EQUAL-TIME CURRENT-CURRENT CORRELATIONS IN NUCLEONS FROM ELECTRON SCATTERING, T. D. Cohen and D. K. Griegel, U. Md. PP\#94-091, DOE/ER/40762029, submitted to Phys. Lett.

5. STRANGER IN THE LIGHT: THE STRANGE VECTOR FORM FACTORS OF THE NUCLEON, H. Forkel, M. Nielsen, X. Jin and T. D. Cohen, U. Md. PP\#94-097, DOE/ER/40762-030, submitted to Phys. Rev. D.

6. QCD SUM RULES FOR $\sum$ HYPERONS IN NUCLEAR MATTER, X. Jin and M. Nielsen, U. Md. PP\#94-118, DOE/ER/40762-032, submitted to Phys. Rev. C.

7. PENGUINS LEAVING THE POLE: BOUND-STATE EFFECTS IN $B \rightarrow K^{*} \gamma$, C. E. Carlson and J. Milana, U. Md. PP\#94-129, DOE/ER/40762-033, submitted to Phys. Rev. D.

8. TESTING QCD SUM RULE TECHNIQUES ON THE LATTICE, D. B. Leinweber, U. Md. PP\#94-130, DOE/ER/40762-034, submitted to Phys. Rev. D.

9. COMMENSURATE SCALE RELATIONS IN QUANTUM CHROMODYNAMICS, S. J. Brodsky and H. J. Lu, U. Md. PP\#94-135, DOE/ER/40762-036, submitted to Phys. Rev. D.

10. RELATIONS AMONG ZERO MOMENTUM CORRELATORS FOR HEAVY-LIGHT SYSTEMS IN QCD, X. Jin and T. D. Cohen, U. Md. PP\#94-142, DOE/ER/40762-038, submitted to Phys. Rev. D.

11. EXCLUSIVE PRODUCTION OF HIGGS BOSONS IN HADRON COLLIDERS, H. J. Lu and J. Milana, PP\#94-177, DOE/ER/40762-041, submitted to Phys. Rev. D. 
12. CONSTRAINTS ON THE FINITE-DENSITY SPECTRAL SPECTRAL DENSITIES OF VECTOR CHANNEL, X. Jin, PP\#94-167, DOE/ER/40762-039, submitted to Phys. Rev. C.

13. VANISHING CONDENSATES AND ANOMALOUSLY LIGHT GOLDSTONE MODES IN MEDIUM, T. D. Cohen and W. Broniowski, PP\#95-008, DOE/ER/40762-043, submitted to Phys. Rev. Lett.

Theses: 1993-94

1. MESON BOUND STATES WITH RELATIVISTIC PHENOMENOLOGICAL $\bar{q} q$ POTENTIALS, C. Edward Bell (1993) (Advisor: S. J. Wallace)

2. A QCD DESCRIPTION OF BARYON SELF-ENERGIES IN NUCLEAR MATTER, Xuemin Jin (1993) (Advisor: Thomas D. Cohen) 